\title{
Probiotics Bacillus licheniformis Improves Intestinal Health of Subclinical Necrotic Enteritis-Challenged Broilers
}

\section{OPEN ACCESS}

Edited by:

Jing Wang,

Institute of Animal Husbandry

and Veterinary Medicine, Beijing

Academy of Agriculture and Forestry

Sciences, China

Reviewed by:

Nikola Puvaèa,

University Business Academy in Novi

Sad, Serbia

Rifat Ullah Khan,

University of Agriculture, Faisalabad,

Pakistan

Jesica Blajman

CONICET Santa Fe, Argentina

Tagang Aluwong,

Ahmadu Bello University, Nigeria

Jianping Wang,

Sichuan Agricultural University, China

*Correspondence:

Zhong Wang

wangzh@cau.edu.cn

${ }^{\dagger}$ These authors have contributed equally to this work

Specialty section:

This article was submitted to

Systems Microbiology,

a section of the journal

Frontiers in Microbiology

Received: 30 October 2020

Accepted: 25 March 2021

Published: 18 May 2021

Citation:

Kan L, Guo F, Liu Y, Pham VH, Guo $Y$ and Wang $Z$ (2021) Probiotics

Bacillus licheniformis Improves

Intestinal Health of Subclinical

Necrotic Enteritis-Challenged Broilers.

Front. Microbiol. 12:623739.

doi: 10.3389/fmicb.2021.623739
Liugang Kan', Fangshen Guo', Yan Liu, Van Hieu Pham, Yuming Guo and Zhong Wang*

State Key Laboratory of Animal Nutrition, College of Animal Science and Technology, China Agricultural University, Beijing, China

Necrotic enteritis infection poses a serious threat to poultry production, and there is an urgent need for searching effective antibiotic alternatives to control it with the global ban on in-feed antibiotics. This study was conducted to investigate the effects of dietary Bacillus licheniformis replacing enramycin on the growth performance and intestinal health of subclinical necrotic enteritis (SNE)-challenged broilers. In total, 504 1-dayold Arbor Acres male chickens were selected and subsequently assigned into three treatments, including PC (basal diet + SNE challenge), PA (basal diet extra $10 \mathrm{mg} / \mathrm{kg}$ enramycin + SNE challenge), and PG (basal diet extra $3.20 \times 10^{9}$ and $1.60 \times 10^{9} \mathrm{CFU}$ B. licheniformis per $\mathrm{kg}$ diet during 1-21 days and 22-42 days, respectively $+\mathrm{SNE}$ challenge). Results showed that $B$. licheniformis significantly decreased the intestinal lesion scores and down-regulated the Claudin-3 mRNA levels in jejunum of SNEinfected broilers on day 25, but increased the mucin-2 gene expression in broilers on day 42. In addition, $B$. licheniformis significantly up-regulated the mRNA levels of TRIF and NF-kB of SNE-challenged broilers compared with the control group on day 25 and TLR-4, TRIF compared with the control and the antibiotic group on day 42. The mRNA expression of growth factors (GLP-2 and TGF- $\beta 2$ ) and HSPs (HSP60, HSP70, and HSP90) were up-regulated in B. licheniformis supplementary group on days 25 and 42 compared with group PC. LEfSe analysis showed that the relative abundance of Lachnospiraceae_UCG_010 was enriched in the PG group; nevertheless, Clostridiales_vadinBB60 and Rnminococcaceae_NK4A214 were in PA. PICRUSt analysis found that the metabolism of cofactors and vitamins, amino acid metabolism, and carbohydrate metabolism pathways were enriched, whereas energy metabolism, membrane transport, cell motility, and lipid metabolism were suppressed in B. licheniformis-supplemented groups as compared with the PC control. In conclusion, dietary supplementation of $B$. licheniformis alleviated the intestinal damage caused by SNE challenge that coincided with modulating intestinal microflora structure and barrier function as well as regulating intestinal mucosal immune responses.

Keywords: subclinical necrotic enteritis, Bacillus licheniformis, intestinal health, immune response, microflora, broiler chicken 


\section{INTRODUCTION}

Necrotic enteritis (NE) is an intestinal bacterial disease in poultry caused by Clostridium perfringens infection and annually costs up to six billion US dollars in production globally (Wade and Keyburn, 2015). C. perfringens is a spore-forming, strictly anaerobic Gram-positive bacterium which could produce up to 17 kinds of toxins (Parish, 1961). According to the secreted toxins, C. perfringens can be divided into five types: types $\mathrm{A}, \mathrm{B}, \mathrm{C}, \mathrm{D}$, and $\mathrm{E}$. NE was caused by $C$. perfringens type A and/or C infection (Engström et al., 2003; Prescott et al., 2016). NE is typically divided into clinical NE and subclinical NE. Clinical NE usually exhibits mass death with a mortality rate up to $50 \%$ and causes intestinal ulcer erosion, bloody feces, and so on (Lee et al., 2011; Alnassan et al., 2014). However, SNE leads to mild intestinal damage in the flock, resulting in inappetence, malabsorption, poor digestion, and further impaired growth performance with a mortality generally less than 5\% (Timbermont et al., 2011). Therefore, chronic intestinal mucosal damage in SNE-infected broilers causes more serious economic losses than clinical NE infections due to the difficulty in detection. Previous studies have demonstrated that $\mathrm{NE}$ infection is usually accompanied by intestinal lesions in broilers, disorders in intestinal microflora (Latorre et al., 2018), intestinal inflammation (Collier et al., 2008; Park et al., 2008), and damages of intestinal tight junction and mucus barrier in broilers (Golder et al., 2011; Forder et al., 2012; Guo et al., 2014). Therefore, modulation on intestinal health may be a great strategy to control NE infection in broiler.

In the post-antibiotic era, apart from plant extracts (Abudabos et al., 2017; Yin et al., 2017), organic acids (Song et al., 2017), polysaccharides (Tian et al., 2016), and vaccines (Mishra and Smyth, 2017), probiotics had been demonstrated to be an effective measure to promote animal growth (Khan and Naz, 2013; Mingmongkolchai and Panbangred, 2018). Evidences indicated that probiotics were one of the effective methods to prevent SNE infection in poultry for its protection on intestinal health (Venessa et al., 2016; Wang Y. et al., 2017). Bacillus licheniformis is a Gram-positive bacterium and characterized by high temperature and stress resistance. Previous studies had found that $B$. licheniformis could produce a variety of biologically active substances, such as digestive enzymes, lysozyme, bacteriocin, and antibacterial peptides, which promote animal performance by improving feed digestibility, stimulating the development of immune system, enhancing intestinal mucosal barrier function, inhibiting the colonization of pathogenic bacteria, promoting the proliferation of potentially beneficial microorganisms, and maintaining the balance of intestinal microflora (Rozs et al., 2001; Kim et al., 2004; Zhou et al., 2016). For example, Wang Y. et al. (2017) reported that B. licheniformis up-regulated the gene expression of tight junction proteins (TJP) and mucin2 in laying hens, thus maintaining the intestinal mechanical barrier and reducing intestinal permeability. Other research noted that probiotics Bacillus spp. strengthened host intestinal mucosal immunity through increasing the mRNA expression levels of TLRs, associated downstream adaptor proteins, and $N F-\kappa B$ in broiler chickens (Rajput et al., 2017), up-regulating the mRNA levels of cytokines and sIgA (Baikui et al., 2016). In addition, diets supplemented with $B$. licheniformis could also modulate the composition and structure of intestinal microbiota in broiler chickens challenged with NE (Lin et al., 2017; Xu et al., 2018). Some researchers have confirmed that probiotics Bacillus spp. as feed additives had achieved promising results in preventing and controlling NE infection in poultry (Jayaraman et al., 2013; Zhou et al., 2016; Wu et al., 2018). However, probiotic strains differ regarding their properties and clinical effects that they elicit; these differences are even observed when the strains belong to the same bacterial species. Therefore, the aim of this study was to explore whether B. licheniformis could alleviate the SNE infection similar to enramycin and reveal its action mechanism by determining intestinal barrier function, the immune responses as well as intestinal microflora.

\section{MATERIALS AND METHODS}

\section{Experimental Animals, Diets, and Treatments}

A total of 504 1-day-old male Arbor Acres chicks with an average weight at $43.9 \mathrm{~g}$ (SD 0.87) were purchased from Beijing Arbor Acres Poultry Breeding Company (Beijing, China). On arrival, chicks were weighed and randomly assigned to three groups. Each group contained 12 replicates with 14 birds per replicate. Each replicate was reared in a separate isolator $\left(240 \times 60 \times 60 \mathrm{~cm}^{3}\right)$. The treatment groups were as follows: (1) positive control group (PC, basal diet + SNE infection); (2) antibiotic group (PA, basal diet extra $10 \mathrm{mg} / \mathrm{kg}$ enramycin + SNE infection); (3) B. licheniformis-treated group (PG, basal diet extra $3.20 \times 10^{9} \mathrm{CFU}$ B. licheniformis per $\mathrm{kg}$ diet [days 1-21], $1.60 \times 10^{9} \mathrm{CFU}$ B. licheniformis per $\mathrm{kg}$ diet [days 21-42] + SNE infection). B. licheniformis used in this study was provided by Chr. Hansen Co., Ltd. (Denmark) at a density of $3.20 \times 10^{9} \mathrm{CFU} / \mathrm{g}$. Antibiotic-free and coccidiostatfree corn-soybean meal basal diets were formulated according to National Research Council (1994) requirements for starter (days 1 to 21 ) and grower (days 22 to 42 ) periods. The composition and nutrient levels of the basal diet are presented in Table 1. The experimental diet was formulated by mixing the basal diet with $B$. licheniformis to reach $3.20 \times 10^{9}$ and $1.60 \times 10^{9} \mathrm{CFU} / \mathrm{kg}$ of diet in the starter and grower periods, respectively. To ensure the homogeneity of the additives, approximately $5 \mathrm{~kg}$ of the basal diet mixed with the additive was thoroughly mixed using a plastic bucket. Starter diets were pelleted and crumbled, whereas grower diets were just pelleted. All birds were reared in a farm and fed ad libitum and allowed to access water freely throughout the entire experimental period. Room temperature was maintained at $33^{\circ} \mathrm{C}$ during first 5 days and then gradually decreased by $2^{\circ} \mathrm{C}$ weekly until a final room temperature of $24^{\circ} \mathrm{C}$ was reached. Artificial light was provided in a $23 \mathrm{~h}$ light $/ 1 \mathrm{~h}$ dark program. In addition, all birds were immunized with Newcastle disease virus vaccine plus infectious bronchitis virus vaccine via drinking water on day 21 . 


\section{Experimental Induction of SNE}

Avian C. perfringens type A strain CVCC2030 (China Veterinary Culture Collection Center, Beijing, China) was used for infection in this study. C. perfringens was anaerobically cultured in thioglycolate broth for $24 \mathrm{~h}$ at $37^{\circ} \mathrm{C}$, then aseptically transferred into a cooked meat medium (CM605; Beijing Land Bridge Technology Co., Ltd.) supplemented with dried meat particles (CM607; Beijing Land Bridge Technology Co., Ltd.) and iron powders (Shanghai Kefeng Industry \& Commerce Co., Ltd.) and incubated anaerobically for $18 \mathrm{~h}$ at $37^{\circ} \mathrm{C}$. Establishment of SNE model in broilers referenced $\mathrm{Wu}$ et al. (2018) with little modifications. Briefly, each bird in this study was orally gavaged with 12,000 Eimeria maxima oocysts (College of Veterinary Medicine, China Agricultural University, Beijing, China) at 12 days of age, and subsequently with $1 \mathrm{ml}$ of $C$. perfringens $\left(1 \times 10^{9} \mathrm{CFU} / \mathrm{ml}\right)$ once a day during days 17 to 23 to establish the SNE model.

\section{Growth Performance}

On days 21 and 42, the body weight (BW) and feed intake of each replicate were recorded. Then the average gain

TABLE 1 | Composition and nutrient levels of the basal diets.

\begin{tabular}{|c|c|c|}
\hline Items & Weeks 0-3 & Weeks 4-6 \\
\hline \multicolumn{3}{|l|}{ Ingredient, \% } \\
\hline Corn (CP 7.8\%) & 37.15 & 55.33 \\
\hline Wheat middlings & 0 & 5.00 \\
\hline Wheat & 20.00 & 0 \\
\hline Soybean meal (CP 46.8\%) & 34.00 & 31.00 \\
\hline Soybean oil & 4.80 & 5.00 \\
\hline Limestone & 0.90 & 0.70 \\
\hline Dicalcium phosphate & 2.00 & 2.00 \\
\hline DL-Methionine, 98\% & 0.23 & 0.19 \\
\hline L-Lysine sulfate, $78 \%$ & 0.15 & 0.10 \\
\hline Sodium chloride & 0.30 & 0.30 \\
\hline Ethoxyquinoline, 33\% & 0.05 & 0.05 \\
\hline Choline chloride, $50 \%$ & 0.24 & 0.15 \\
\hline Vitamin premix ${ }^{a}$ & 0.03 & 0.03 \\
\hline Mineral premix $x^{b}$ & 0.15 & 0.15 \\
\hline Total & 100 & 100 \\
\hline \multicolumn{3}{|l|}{ Nutrient levels } \\
\hline Metabolizable energy, Mcal/kg & 3.03 & 3.10 \\
\hline Crude protein, \% & 21.77 & 19.80 \\
\hline Calcium, \% & 1.06 & 0.95 \\
\hline Non-phytate, \% & 0.45 & 0.42 \\
\hline Lysine, \% & 1.23 & 1.10 \\
\hline Methionine, \% & 0.52 & 0.46 \\
\hline Met + Cys, \% & 0.83 & 0.74 \\
\hline
\end{tabular}

a Vitamin premix provided per kilogram of complete diet: vitamin A 12,500 IU; vitamin $D_{3} 2500 \mathrm{IU}$; vitamin E $30 \mathrm{IU}$; vitamin $K_{3} 2.65$ mg; vitamin $B_{12} 0.025$ mg; biotin 0.0325 mg; folic acid 1.25 mg; nicotinic acid 50 mg; pantothenic acid 12 mg; riboflavin $6 \mathrm{mg}$; thiamine mononitrate $2 \mathrm{mg}$.

${ }^{b}$ Mineral premix provided per kilogram of complete diet: iron $80 \mathrm{mg}$; copper $8 \mathrm{mg}$; manganese 100 mg; zinc 75 mg; iodine 0.35 mg; selenium 0.15 mg.
(AG), feed intake (FI), and feed conversion ratio (FCR) were calculated for days 1-21, 22-42, and 1-42, respectively. Death of birds in each replicate was recorded daily and was used for determining the mortality.

\section{Intestinal Lesion Score and Sample Collection}

On days 25 and 42, one bird per replicate was randomly selected, weighed, and euthanized by jugular exsanguination. The middle segments of jejunum (approximately $1 \mathrm{~cm}$ ) were cut off carefully and gently rinsed with ice-cold sterile saline to remove internal digesta. Subsequently, the jejunum segments were fixed in $4 \%$ paraformaldehyde immediately for further morphology analysis. Another jejunum sample was collected and washed, then frozen in liquid nitrogen immediately and stored at $-80^{\circ} \mathrm{C}$ for the subsequent gene expression analysis. Liver and cecal digesta samples were put into sterile tubes, snap-frozen in liquid nitrogen, and transferred to $-80^{\circ} \mathrm{C}$. Liver samples were used to determine microbial translocation, while cecal samples were used to determine bacterial populations, short-chain fatty acid (SCFA) contents, and DNA extraction. The duodenum, jejunum, and ileum of each bird were cut longitudinally and scored 0 (none) to 4 (severe) for NE gut lesions by three independent observers blindly as previously described by Gholamiandehkordi et al. (2007) with some modifications.

The scoring criteria are as follows: $0=$ no obvious lesions; $1=$ thin and friable intestine with hemorrhagic spots (1-5 foci); 2 = small gas production and focal necrosis or ulceration (hemorrhagic spots 6-15); 3 = gas-filled intestine and patches of necrosis 1 to $2 \mathrm{~cm}$ long; 4 = diffuse necrosis with great amounts of gas in the intestine.

\section{Bacterial Population of Cecal Digesta and Liver Bacterial Translocation}

Quantification of bacterial population in cecal digesta (or liver) was done with techniques as previously described ( $\mathrm{Wu}$ et al., 2018). Briefly, approximately $1 \mathrm{~g}$ of each sample was diluted with $9 \mathrm{ml}$ ice-cold sterile buffered peptone water (CM201; Land Bridge Technology Ltd.) and homogenized. The homogenized suspension of each sample was serially diluted up to $10^{-7}$, then $100 \mu$ l of each dilution was plated on selective ager plates for bacterial quantification. Each sample was plated in duplicate. Commercial media were used for cultivation of $C$. perfringens (tryptose-sulfite-cycloserine agar, TSC, CM 138; Beijing Land Bridge Technology Co., Ltd.), coliform bacteria (Eosin-Methylene Blue Agar, EMB, CM105; Beijing Land Bridge Technology Co., Ltd.) and lactic acid bacteria (de Man, Rogosa, and Sharpe agar, MRS, CM 188; Land Bridge Technology Co., Ltd.). C. perfringens and lactic acid bacteria were incubated anaerobically for $48 \mathrm{~h}$ at $37^{\circ} \mathrm{C}$, while coliform bacteria were incubated aerobically for $24 \mathrm{~h}$ at $37^{\circ} \mathrm{C}$. The number of colony-forming units was expressed as a logarithmic transformation per gram of cecal digesta (or liver). 


\section{Intestinal Morphology Observation and Analysis}

Fixed jejunum tissues were embedded in paraffin, then sliced into $5-\mu \mathrm{m}$ thickness, deparaffinized in xylene, rehydrated, and mounted on glass slides. Periodic acid-Schiff (PAS) stain was used to stain the sections for determining the number of goblet cells, whereas H\&E stain was used for villous morphology measurement. Five intact villi in every slide were chosen for measurement of goblet cells, villus height $(\mathrm{VH})$, and crypt depth (CD) with Image-pro plus 6.0 (Media Cybernetics, Inc., Rockville, $\mathrm{MD}$, United States) at $\times 40$ magnification. The means of villus height and crypt depth were calculated and subsequently were used to obtain the $\mathrm{VH} / \mathrm{CD}$.

\section{Gene Expression in Jejunum}

Extraction of total RNA in jejunum was performed using Trizol reagent (Invitrogen Life Technologies, Carlsbad, CA, United States) according to the manufacturer's instructions. The concentration and purity of total RNA were determined by using a NanoDrop-2000 spectrophotometer (Thermo Fisher Scientific, Waltham, MA, United States). Then, complementary DNA (cDNA) was synthesized by using Primer Script RT Reagent kit (Takara Bio Inc.) according to the manufacturer's instructions. Using the synthesized cDNA as a template, quantitative realtime PCR (qRT-PCR) was performed in Applied Biosystems' 7500 Fast Real-Time PCR System with SYBR Premix Ex Taq kit (Takara Bio Inc.) in accordance with the manufacturer's guidelines. Thermocycling protocol was as follows: $95^{\circ} \mathrm{C}$ for $30 \mathrm{~s}$, followed by 40 cycles of $95^{\circ} \mathrm{C}$ for $5 \mathrm{~s}$ and $60^{\circ} \mathrm{C}$ for $34 \mathrm{~s}$ for denaturation and annealing/extension, respectively. The purity and specificity of PCR products were determined by melt curve analysis. All data were analyzed using the $2^{-\Delta \Delta C t}$ method, and glyceraldehyde 3-phosphate dehydrogenase (GAPDH) and $\beta$-actin were used to normalize the relative mRNA levels (Livak and Schmittgen, 2001). All samples $(n=6)$ from each group on days 25 and 42 were done in triplicate. Target genes include TJP genes (Occludin, Claudin-1, Claudin-3, Zonula occludens-1[ZO1], mucin-2), TLR signal pathway-related genes (TLR-4, TLR-2, TRIF, MyD88, NF-кB, IL-1 $\beta, I L-10, I L-17, I F N-\gamma, T N F-\alpha)$, heat shock protein genes (HSP60, HSP70, HSP90), and growth factor genes (IGF-2, GLP-2, TGF- $\beta 2$ ). Primers of target genes used in this study are presented in Supplementary Tables 1, 2.

\section{SCFA Concentration in Cecal Content}

A total of $0.5-1.0 \mathrm{~g}$ cecal digesta from day 42 sample was weighed and put into a $10-\mathrm{ml}$ polypropylene tube with $8 \mathrm{ml}$ deionized water, then an ultrasonic bath was performed for $30 \mathrm{~min}$, the suspension subsequently was centrifuged at $8000 \mathrm{rpm}$ for $10 \mathrm{~min}$. The supernatant was collected and diluted 10 fold, and then filtered with a $0.22-\mu \mathrm{m}$ filtrator. Next, $25 \mu \mathrm{l}$ of filtered solution was subjected to high-performance ion chromatography system (ICS-3000; Dionex, United States) for conductivity detection analysis. Organic acids were separated on an AS11 analytical column $\left(250 \times 4 \mathrm{~mm}^{2}\right)$ and an AG11 guard column under the following gradient conditions (the gradient was based on potassium hydroxide): $0-5 \mathrm{~min}, 0.8-1.5 \mathrm{mM}$; $5-$ $10 \mathrm{~min}, 1.5-2.5 \mathrm{mM}$; and $10-15 \mathrm{~min}, 2.5 \mathrm{mM}$; the flow rate was $1.0 \mathrm{ml} / \mathrm{min}$. The results of SCFAs were expressed as milligrams per kilogram of digesta.

\section{Microbial DNA Extraction, 16S rRNA Gene Amplification, Sequencing, and Bioinformatics Analysis}

Bacterial DNA extraction of day 25 cecal digesta was performed by using PowerSoil DNA Isolation Kit (ANBIOSCI Tech Ltd., United States) according to the manufacturer's instructions. Integrity of DNA was appraised by agarose gel electrophoresis, then the qualified DNA was used as template for the V3-V4 region of bacterial $16 \mathrm{~S}$ rRNA gene amplification with barcoded primer pair 338F: 5'-ACTCCTACGGGAGGCAGCA-3' and 806R: 5'-GGACTACHVGGGTWTCTAAT-3'. The KAPA HiFi Hotstart ReadyMix PCR kit (Kapa Biosystems, United States) was used in the PCR amplification and the procedures were as follows: $98^{\circ} \mathrm{C}$ for $2 \mathrm{~min}(1 \mathrm{cycle}), 98^{\circ} \mathrm{C}$ for $30 \mathrm{~s} / 50^{\circ} \mathrm{C}$ for $30 \mathrm{~s} / 72^{\circ} \mathrm{C}$ for $1 \mathrm{~min}(25 \mathrm{cycles})$, and finally $72^{\circ} \mathrm{C}$ lasts for $5 \mathrm{~min}$. The amplification products were determined by $2 \%$ agarose gel and purified with AxyPrep DNA Gel Extraction Kit (Axygen Biosciences, Union City, CA, United States). Amplicon libraries were sequenced on Illumina HiSeq 2500 platform (Illumina, San Diego, CA, United States) at Biomarker Technologies Co., Ltd. (Beijing, China). The sequencing data were merged using FLASH (version 1.2.11) to get raw tags. Raw tags were then subjected to filtration (Trimmomatic, version 0.33) and chimera sequences removed (UCHIME, version 8.1) to obtain effective tags. UCLUST (Edgar, 2010) was used to cluster effective tags into operational taxonomic units (OTUs) at a similarity level of $97 \%$ with QIIME software (version 1.8.0) (Caporaso et al., 2010). Afterward, basing on the Silva taxonomic database, OTUs were annotated. Venn diagram, rarefaction curve, and bacteria relative abundance were created with $\mathrm{R}$ software (version 2.15.3). Alpha diversity, including ACE, Chaol, Simpson, and Shannon index, were investigated by Mothur (version 1.30), and the significance of these items was determined using a Mann-Whitney $U$ test. $\beta$ Diversity was calculated from binary_jaccard distance (PERMANOVA/ANOSIM analysis) in QIIME software. A twosided Student's $t$-test was used to determine the significance of the differences between groups. Line discriminant analysis (LDA) effect size (LEfSe ${ }^{1}$ ) (Segata et al., 2011) tool was used to determine statistically different biomarkers between groups (LDA value: 2) based on the taxonomic files obtained from the QIIME analysis. The raw sequences used in our study had been uploaded at the Sequence Read Archive of the National Center for Biotechnology Information, with the study accession number PRJNA574872. The functions of the cecum metagenomes were predicted using PICRUSt (Phylogenetic investigation of communities by reconstruction of unobserved states) analysis based on highquality sequences (Langille et al., 2013).

\footnotetext{
${ }^{1}$ http://huttenhower.sph.harvard.edu/lefse/
} 


\section{Statistical Analysis}

All results were displayed as means \pm SEM. Statistical significance of growth performance, intestinal lesion scores, bacterial population, intestinal morphology, gene expression, and SCFA content were determined by one-way ANOVA, followed by Duncan's multiple comparison test (SPSS, version 20.0, Chicago, IL, United States). Kruskal-Wallis test was employed to analyze the difference in bacterial relative abundance. Significant difference was declared when $P<0.05$.

\section{RESULTS}

\section{Growth Performance}

We measured four indexes concerning broiler chickens' productivity as shown in Table 2 . There was no significant difference in BW, AG, FI, and FCR between groups, while numerically higher BW, AG, and lower FCR were observed in PA and PG groups when compared with PC group at days 21-42 and days 1-42, and the value of those indexes of PA and PG group were close to each other. There was no significant difference in mortality among groups.

\section{Small Intestine Lesion Scores and Jejunum Morphology}

As shown in Table 3, lesion scores of duodenum and small intestine in PG group were significantly lower than those in PC and PA group and jejunum lesion score in PG group was

TABLE 2 | Effects of $B$. licheniformis and enramycin on growth performance of broilers challenged with SNE.

\begin{tabular}{lccccc}
\hline Items & PC & PA & PG & SEM & $P$ values \\
\hline Days 1-21 & & & & & \\
BW (g/bird) & 641 & 652 & 640 & 5.7 & 0.623 \\
AG (g/bird) & 598 & 609 & 597 & 5.7 & 0.624 \\
Fl (g/bird) & 856 & 866 & 853 & 6.4 & 0.694 \\
FCR & 1.43 & 1.42 & 1.43 & 0.005 & 0.631 \\
Mortality (\%) & 0.00 & 1.19 & 0.60 & 0.439 & 0.555 \\
Days 21-42 & & & & & \\
BW (g/bird) & 2346 & 2394 & 2395 & 11.1 & 0.103 \\
AG (g/bird) & 1705 & 1734 & 1753 & 10.8 & 0.178 \\
FI (g/bird) & 2996 & 2991 & 3036 & 14.4 & 0.384 \\
FCR & 1.76 & 1.73 & 1.73 & 0.008 & 0.221 \\
Mortality (\%) & 1.19 & 1.19 & 0.60 & 0.418 & 0.807 \\
Days 1-42 & & & & & \\
BW (g/bird) & 2346 & 2394 & 2395 & 11.1 & 0.103 \\
AG (g/bird) & 2303 & 2351 & 2352 & 11.1 & 0.103 \\
Fl (g/bird) & 3852 & 3865 & 3892 & 16.8 & 0.614 \\
FCR & 1.67 & 1.64 & 1.65 & 0.006 & 0.101 \\
Mortality (\%) & 1.19 & 2.38 & 1.19 & 0.577 & 0.636 \\
\hline
\end{tabular}

Data are presented as means \pm SEM ( $n=12$; cage was used as experimental unit). $P C$, basal diet + SNE; PA, basal diet extra antibiotics + SNE; PG, basal diet extra B. licheniformis + SNE.

BW, body weight; AG, average gain; FI, feed intake; FCR, feed conversion rate (FI/AG).
TABLE 3 | Effects of $B$. licheniformis and enramycin on intestinal lesion scores of broilers challenged with SNE.

\begin{tabular}{lccccc}
\hline Items & PC & PA & PG & SEM & P values \\
\hline Day 25 & & & & & \\
Duodenum & $1.13^{\mathrm{a}}$ & $1.08^{\mathrm{a}}$ & $0.38^{\mathrm{b}}$ & 0.081 & 0.000 \\
Jejunum & $0.75^{\mathrm{ab}}$ & $1.04^{\mathrm{a}}$ & $0.67^{\mathrm{b}}$ & 0.060 & 0.022 \\
lleum & 0.29 & 0.38 & 0.08 & 0.061 & 0.097 \\
Small intestine & $2.17^{\mathrm{a}}$ & $2.50^{\mathrm{a}}$ & $1.13^{\mathrm{b}}$ & 0.927 & 0.000 \\
Day 42 & & & & & \\
Duodenum & 0.58 & 0.42 & 0.50 & 0.049 & 0.378 \\
Jejunum & 0.29 & 0.29 & 0.25 & 0.051 & 0.856 \\
lleum & 0.04 & 0.00 & 0.13 & 0.027 & 0.147 \\
Small intestine & 0.92 & 0.71 & 0.88 & 0.080 & 0.541 \\
\hline
\end{tabular}

Data are presented as means \pm SEM ( $n=12$; cage was used as experimental unit). $P C$, basal diet + SNE; PA, basal diet extra antibiotics + SNE; $P G$, basal diet extra $B$. licheniformis + SNE. Means within a row lacking a common superscript differ significantly $(P<0.05)$.

TABLE 4 | Effects of $B$. licheniformis and enramycin on jejunal morphology of 25-day-old broilers challenged with SNE.

\begin{tabular}{lccccc}
\hline Items & PC & PA & PG & SEM & P values \\
\hline Goblet cells & $194.8^{\mathrm{a}}$ & $149.5^{\mathrm{b}}$ & $204.6^{\mathrm{a}}$ & 8.196 & 0.005 \\
$\mathrm{VH}(\mu \mathrm{m})$ & 706.8 & 692.3 & 738.1 & 30.014 & 0.828 \\
$\mathrm{CD}(\mu \mathrm{m})$ & 180.5 & 191.8 & 196.4 & 6.964 & 0.953 \\
$\mathrm{VH} / \mathrm{CD}$ & 4.12 & 3.65 & 3.68 & 0.159 & 0.459
\end{tabular}

Data are presented as means \pm SEM ( $n=6$; cage was used as experimental unit). $P C$, basal diet + SNE; PA, basal diet extra antibiotics + SNE; PG, basal diet extra $B$. licheniformis + SNE. Means within a row lacking a common superscript differ significantly $(P<0.05)$.

Goblet cells, the number of goblet cells on a villus; VH, villus height; $C D$, crypt depth.

lower than PA group on 2 days post-infection (DPI) $(P<0.05)$. Similarly, there was a tendency that lesion scores of ileum in PG were lower than in PC and PA groups $(0.05<P<0.1)$. In addition, lesion scores of duodenum, jejunum, and small intestine in PA group were comparable with PC group. At 42 days of age (19 DPI), no significant difference was detected of intestinal lesion scores in the three experiment groups. We also investigated the jejunum morphology of broilers at 2 DPI (Table 4). It was found that the number of goblet cells were significantly decreased in PA group compared with PC and PG group $(P<0.05)$. However, the jejunum villus height $(\mathrm{VH})$, crypt depth $(\mathrm{CD})$, and $\mathrm{VH} / \mathrm{CD}$ values were not changed significantly among groups.

\section{Cecal Bacterial Population and Liver Clostridium perfringens Translocation}

Table 5 shows the results of cecal bacterial population and liver C. perfringens translocation. At 25 days of age (2 DPI), SNE-infected birds fed diets supplemented with enramycin exhibited significantly reduced population of C. perfringens in cecum and liver in contrast to that in PC and PG groups $(P<0.05)$. However, probiotic supplementation failed to decrease the population of $C$. perfringens in both cecum and 
TABLE 5 | Effects of B. licheniformis and enramycin the amounts of bacteria (Ig $\mathrm{CFU} / \mathrm{g}^{1}$ ) in cecal digesta of broilers challenged with SNE.

\begin{tabular}{|c|c|c|c|c|c|c|}
\hline & Items & PC & PA & $P G$ & SEM & $P$ values \\
\hline \multicolumn{7}{|l|}{ Day 25} \\
\hline \multirow[t]{3}{*}{ Cecal digesta } & Clostridium perfringens & $4.62^{\mathrm{a}}$ & $1.75^{\mathrm{b}}$ & $4.11^{a}$ & 0.423 & 0.004 \\
\hline & Escherichia coli & 6.67 & 7.51 & 6.52 & 0.229 & 0.166 \\
\hline & Lactobacillus & 10.15 & 9.83 & 9.48 & 0.222 & 0.495 \\
\hline Liver & Clostridium perfringens & $1.56^{\mathrm{a}}$ & $0.17^{b}$ & $1.46^{\mathrm{a}}$ & 0.233 & 0.011 \\
\hline \multicolumn{7}{|l|}{ Day 42} \\
\hline \multirow[t]{3}{*}{ Cecal digesta } & Clostridium perfringens & 1.24 & 1.53 & 0.90 & 0.270 & 0.657 \\
\hline & Escherichia coli & $6.75^{\mathrm{b}}$ & $7.73^{a}$ & $6.83^{\mathrm{b}}$ & 0.154 & 0.007 \\
\hline & Lactobacillus & 8.69 & 8.16 & 8.26 & 0.178 & 0.469 \\
\hline Liver & Clostridium perfringens & 0.38 & 0.17 & 0.27 & 0.127 & 0.804 \\
\hline \multicolumn{7}{|c|}{$\begin{array}{l}\text { Data are presented as means } \pm S E M(n=6 \text {; cage was used as experimental unit). } \\
P C \text {, basal diet }+S N E ; P A \text {, basal diet extra antibiotics }+S N E ; P G \text {, basal diet extra } \\
\text { B. licheniformis }+S N E \text {. Means within a row lacking a common superscript differ } \\
\text { significantly }(P<0.05) \text {. } \\
{ }^{1} \mathrm{lg} C F U / g \text {, log } 10 \text { colony-forming units per gram of cecal digesta. }\end{array}$} \\
\hline
\end{tabular}

liver. Furthermore, no significant differences were observed in Escherichia coli and Lactobacilli population in cecum between PC, PA, and PG groups. At 42 days of age, the cecal Escherichia coli population in the PA group was significantly higher than that in the PC and PG groups $(P<0.05)$. Similarly, the difference between PG and PC group was not statistically significant.

\section{Jejunal Tight Junction Protein Gene and Mucin Gene Expression}

As depicted in Table 6, the expression of Claudin-3 in the jejunum of SNE-infected chickens fed with $B$. licheniformis was lower than that in the PC group at day $25(P<0.05)$, but no significant difference was seen between PG and PA group. Only a downtrend of Claudin-3 levels was detected in PA group when compared with PC group. At 42 days of age, Claudin-3 mRNA levels in PA group were significantly up-regulated when compared with the PC and PG groups $(P<0.05)$. Besides, a tendency was detected that the ZO-1 expression of PA and PG group was higher than PC group $(0.05<P<0.1)$. Similar with the results of the number of goblet cells on villus, mucin2 expression levels in the PA and PG groups were significantly increased when compared with the PC group at day $42(P<0.05)$. Moreover, the gene expression of mucin-2 in the PG group was much higher than that in the antibiotic supplemented group $(P<0.05)$.

\section{Jejunal Toll-Like Receptors Signaling Pathway and Immune-Related Cytokine Gene Expression}

Table 7 presents the results of jejunal TLRs signaling pathway and immune-related cytokine gene expression in broilers. On
TABLE 6 | Effects of $B$. licheniformis and enramycin on tight junction protein and mucin-2 gene expression in jejunum of broilers challenged with SNE.

\begin{tabular}{lccccc}
\hline Items & PC & PA & PG & SEM & $P$ values \\
\hline Day 25 & & & & & \\
Occludin & 1.03 & 0.82 & 0.83 & 0.048 & 0.128 \\
Claudin-1 & 1.08 & 1.05 & 0.87 & 0.081 & 0.555 \\
Claudin-3 & $1.01^{\mathrm{a}}$ & $0.87^{\mathrm{b}}$ & $0.72^{\mathrm{b}}$ & 0.045 & 0.026 \\
ZO-1 & 1.02 & 1.18 & 0.93 & 0.059 & 0.239 \\
Mucin-2 & 1.01 & 0.94 & 1.01 & 0.036 & 0.677 \\
Day 42 & & & & & \\
Occludin & 1.02 & 1.13 & 1.12 & 0.049 & 0.629 \\
Claudin-1 & 1.03 & 1.04 & 0.90 & 0.051 & 0.505 \\
Claudin-3 & $1.01^{\mathrm{b}}$ & $1.48^{\mathrm{a}}$ & $1.10^{\mathrm{b}}$ & 0.073 & 0.008 \\
ZO-1 & 1.01 & 1.22 & 1.23 & 0.044 & 0.064 \\
Mucin-2 & $1.04^{\mathrm{C}}$ & $1.79^{\mathrm{b}}$ & $2.80^{\mathrm{a}}$ & 0.202 & 0.000 \\
\hline
\end{tabular}

Data are presented as means \pm SEM ( $n=6$; cage was used as experimental unit). $P C$, basal diet + SNE; PA, basal diet extra antibiotics + SNE; PG, basal diet extra $B$. licheniformis + SNE. Means within a row lacking a common superscript differ significantly $(P<0.05)$.

ZO, zonula occludens.

TABLE 7 | Effects of $B$. licheniformis and enramycin on TLR signal pathway-related gene expression in jejunum of broilers challenged with SNE.

\begin{tabular}{|c|c|c|c|c|c|}
\hline Items & PC & PA & $P G$ & SEM & $\boldsymbol{P}$ values \\
\hline \multicolumn{6}{|l|}{ Day 25} \\
\hline$T L R-4$ & 1.02 & 1.35 & 1.29 & 0.077 & 0.174 \\
\hline TLR-2 & 1.02 & 1.21 & 1.18 & 0.077 & 0.592 \\
\hline TRIF & $1.00^{b}$ & $1.24^{\mathrm{a}}$ & $1.33^{\mathrm{a}}$ & 0.045 & 0.002 \\
\hline MyD88 & 1.07 & 1.17 & 0.88 & 0.067 & 0.227 \\
\hline$N F-\kappa B$ & $1.01^{b}$ & $1.52^{\mathrm{a}}$ & $1.42^{\mathrm{a}}$ & 0.080 & 0.012 \\
\hline$I L-1 \beta$ & 1.11 & 1.85 & 1.66 & 0.218 & 0.378 \\
\hline IL-10 & 1.07 & 1.19 & 0.90 & 0.094 & 0.475 \\
\hline IL-17 & $1.10^{b}$ & $2.72^{\mathrm{a}}$ & $1.40^{\mathrm{b}}$ & 0.262 & 0.021 \\
\hline$I F N-\gamma$ & 1.05 & 1.12 & 1.05 & 0.089 & 0.932 \\
\hline$T N F-\alpha$ & 1.02 & 1.37 & 1.35 & 0.074 & 0.084 \\
\hline \multicolumn{6}{|l|}{ Day 42} \\
\hline$T L R-4$ & $1.02^{b}$ & $1.04^{b}$ & $1.52^{\mathrm{a}}$ & 0.075 & 0.002 \\
\hline TLR-2 & 1.05 & 0.84 & 1.10 & 0.068 & 0.299 \\
\hline TRIF & $1.02^{b}$ & $0.76^{c}$ & $1.30^{\mathrm{a}}$ & 0.061 & 0.000 \\
\hline MyD88 & 1.01 & 1.09 & 0.98 & 0.045 & 0.590 \\
\hline$N F-\kappa B$ & $1.04^{b}$ & $1.16^{b}$ & $1.48^{a}$ & 0.072 & 0.024 \\
\hline$I L-1 \beta$ & $1.03^{\mathrm{ab}}$ & $0.61^{b}$ & $1.17^{\mathrm{a}}$ & 0.097 & 0.039 \\
\hline IL-10 & 1.07 & 0.74 & 1.04 & 0.092 & 0.276 \\
\hline IL-17 & $0.98^{a}$ & $0.45^{b}$ & $0.99^{a}$ & 0.097 & 0.016 \\
\hline$I F N-\gamma$ & 1.03 & 0.85 & 0.96 & 0.055 & 0.445 \\
\hline$T N F-\alpha$ & 1.04 & 1.13 & 1.35 & 0.066 & 0.151 \\
\hline
\end{tabular}

Data are presented as means $\pm S E M(n=6$; cage was used as experimental unit). $P C$, basal diet + SNE; PA, basal diet extra antibiotics + SNE; PG, basal diet extra B. licheniformis + SNE. Means within a row lacking a common superscript differ significantly $(P<0.05)$.

day 25 , dietary antibiotics and $B$. licheniformis significantly upregulated the mRNA levels of TRIF and NF- $\mathrm{B}$ in jejunum of SNE-infected broiler chickens compared with the PC group $(P<0.05)$. Moreover, the mRNA expression level of $I L-17$ gene was significantly up-regulated in the PA group than that 
TABLE 8 | Effects of $B$. licheniformis and enramycin on recovery protein gene expression in jejunum of broilers challenged with SNE.

\begin{tabular}{lccccc}
\hline Items & PC & PA & PG & SEM & P values \\
\hline Day 25 & & & & & \\
HSP60 & $1.04^{\mathrm{b}}$ & $1.24^{\mathrm{b}}$ & $2.53^{\mathrm{a}}$ & 0.183 & 0.000 \\
HSP70 & 1.03 & 1.17 & 1.19 & 0.046 & 0.309 \\
HSP90 & $1.01^{\mathrm{c}}$ & $1.83^{\mathrm{b}}$ & $2.51^{\mathrm{a}}$ & 0.177 & 0.000 \\
IGF-2 & $1.04^{\mathrm{b}}$ & $1.65^{\mathrm{a}}$ & $1.05^{\mathrm{b}}$ & 0.102 & 0.010 \\
GLP-2 & $1.04^{\mathrm{b}}$ & $1.81^{\mathrm{a}}$ & $2.22^{\mathrm{a}}$ & 0.175 & 0.010 \\
TGF- $\beta 2$ & $1.02^{\mathrm{b}}$ & $1.65^{\mathrm{a}}$ & $1.37^{\mathrm{ab}}$ & 0.097 & 0.018 \\
Day 42 & & & & & \\
HSP60 & $1.03^{\mathrm{b}}$ & $1.13^{\mathrm{b}}$ & $1.58^{\mathrm{a}}$ & 0.075 & 0.001 \\
HSP70 & $1.01^{\mathrm{b}}$ & $0.95^{\mathrm{b}}$ & $1.37^{\mathrm{a}}$ & 0.055 & 0.000 \\
HSP90 & $1.02^{\mathrm{b}}$ & $0.65^{\mathrm{c}}$ & 1.37 & 0.083 & 0.000 \\
IGF-2 & $1.04^{\mathrm{b}}$ & $1.13^{\mathrm{b}}$ & 1.47 & 0.093 & 0.141 \\
GLP-2 & $1.09^{\mathrm{b}}$ & $1.34^{\mathrm{b}}$ & $1.83^{\mathrm{a}}$ & 0.108 & 0.011 \\
TGF- $\beta 2$ & $1.02^{\mathrm{b}}$ & $1.24^{\mathrm{b}}$ & $1.65^{\mathrm{a}}$ & 0.081 & 0.001
\end{tabular}

Data are presented as means \pm SEM ( $n=6$; cage was used as experimental unit). $P C$, basal diet + SNE; PA, basal diet extra antibiotics + SNE; PG, basal diet extra $B$. licheniformis + SNE. Means within a row lacking a common superscript differ significantly $(P<0.05)$.

HSP, heat shock protein; IGF, insulin-like growth factors; GLP, glucagonlike peptide.

in the PC and PG groups $(P<0.05)$. On day 42, jejunal mRNA expression levels of TLR-4, TRIF, and $N F-\kappa B$ were significantly increased in the PG group when compared with the PC and PA groups $(P<0.05)$. Interestingly, birds fed diet supplemented with antibiotics had significantly lower $I L-17$ and TRIF mRNA levels in contrast to the PC group $(P<0.05)$. In addition, supplementation of $B$. licheniformis also significantly up-regulated $I L-1 \beta$ mRNA levels compared with the PA group $(P<0.05)$.

\section{Gene Expression of Jejunal Heat Shock Proteins and Growth Factors}

On day 25, when compared with the PC group, addition of antibiotics in feed significantly up-regulated the relative gene expressions of HSP90, IGF-2, GLP-2, and TGF- $\beta 2$ in the jejunum of SNE-infected broilers $(P<0.05$, Table 8). Meanwhile, higher mRNA levels of HSP60, HSP90, and GLP-2 were also detected in the PG group when compared with the PC group $(P<0.05)$. On day 42 , dietary antibiotics had significantly lower mRNA levels of HSP90 than that in PC group $(P<0.05)$, whereas birds fed diets supplemented with $B$. licheniformis exhibited significantly higher gene expression of HSP60, HSP70, HSP90, GLP-2, and TGF- $\beta 2$ when compared with the PC and PA groups $(P<0.05)$.

\section{Short-Chain Fatty Acids in Cecal Content}

On day 42, we investigated the SCFA concentrations in cecal content of SNE-infected broilers (Table 9). Significant changes were only observed in the formic acid concentration between groups. The concentration of formic acid in the PA and PG groups were significantly increased compared with the PC group
TABLE 9 | Effects of $B$. licheniformis and enramycin on concentration of short-chain fatty acids in cecal content of 42 -day-old broilers $(\mathrm{mg} / \mathrm{kg})$.

\begin{tabular}{lccccc}
\hline Items & PC & PA & PG & SEM & P values \\
\hline Lactic acid & 297.2 & 216.6 & 390.1 & 69.34 & 0.619 \\
Formic acid & $215.7^{\mathrm{b}}$ & $276.1^{\mathrm{a}}$ & $273.9^{\mathrm{a}}$ & 12.74 & 0.027 \\
Acetic acid & 3958.7 & 3886.8 & 3737.9 & 138.68 & 0.822 \\
Propionic acid & 2467.9 & 1843.1 & 2091.2 & 135.81 & 0.170 \\
Butyric acid & 1584.2 & 1284.4 & 1415.1 & 97.46 & 0.480 \\
Isobutyric acid & 61.5 & 52.9 & 69.3 & 6.780 & 0.655 \\
Valeric acid & 121.7 & 101.7 & 125.1 & 6.547 & 0.305 \\
Isovaleric acid & 66.0 & 92.7 & 73.2 & 9.328 & 0.508
\end{tabular}

Data are presented as means \pm SEM ( $n=6$; cage was used as experimental unit). $P C$, basal diet + SNE; PA, basal diet extra antibiotics + SNE; PG, basal diet extra $B$. licheniformis + SNE. Means within a row lacking a common superscript differ significantly $(P<0.05)$.

TABLE 10 | Effects of $B$. licheniformis and enramycin on alpha diversity of cecal microbiota of 25-day-old broilers challenged with SNE.

\begin{tabular}{lccccc}
\hline Items & PC & PA & PG & SEM & $P$ values \\
\hline ACE & 417.1 & 365.8 & 393.1 & 15.123 & 0.407 \\
Chao1 & 423.6 & 384.7 & 397.5 & 19.036 & 0.280 \\
Simpson & 0.115 & 0.126 & 0.101 & 0.019 & 0.870 \\
Shannon & 3.46 & 3.21 & 3.40 & 0.167 & 0.746 \\
\hline
\end{tabular}

Data are presented as means \pm SEM ( $n=6$; cage was used as experimental unit). $P C$, basal diet + SNE; PA, basal diet extra antibiotics + SNE; PG, basal diet extra B. licheniformis + SNE. Means within a row lacking a common superscript differ significantly $(P<0.05)$.

$(P<0.05)$, but no significant differences were detected between $\mathrm{PA}$ and $\mathrm{PG}$ groups $(P>0.05)$.

\section{Cecal Microbiome}

A total of $1,440,717$ pairs of reads were generated after $16 \mathrm{~S}$ rRNA sequencing of 18 cecal digesta samples. Then, we obtained 1,191,203 effective Tags after splicing, filtering, and removal of chimeras, and an average of 66,178 effective Tags were obtained from each sample. Based on $97 \%$ sequence similarity, Tags were clustered into 510 OTUs, of which 475 OTUs were shared by three groups, and only 2, 4, and 1 OTUs were exclusive in PC, PA, and PG groups, respectively (Supplementary Figure 1). Furthermore, the alpha diversity analysis of cecal microbiota showed that no significant difference in ACE, Chao1, Simpson, and Shannon index between groups $(P>0.05$ and Table 10).

The representative sequences of OTUs were annotated with Silva database. Then we analyzed the bacterial composition in phylum and genus level of samples. The most abundant (top 6) phyla of bacteria are presented in Supplementary Figure 2A. At the phylum level, the cecal microbiota was dominated by Firmicutes, Bacteroidetes, Proteobacteria, and Tenericutes, together accounting for over $99.7 \%$ of the total sequences. However, no significant differences were detected in those phyla between groups $(P>0.05$, Table 11). The top 10 abundant bacteria in genus level were Faecalibacterium, Lactobacillus, Barnesiella, [Ruminococcus]_torques_group, 
TABLE 11 | Effects of $B$. licheniformis and enramycin on relative abundances of phyla in cecal microbiota of 25-day-old broilers challenged with SNE (\%).

\begin{tabular}{lccccc}
\hline Items & PC & PA & PG & SEM & $P$ values \\
\hline Firmicutes & 72.68 & 72.41 & 86.55 & 3.751 & 0.203 \\
Bacteroidetes & 23.27 & 20.71 & 10.47 & 3.938 & 0.519 \\
Proteobacteria & 2.20 & 4.61 & 1.01 & 1.012 & 0.325 \\
Tenericutes & 1.68 & 1.84 & 1.70 & 0.388 & 0.805 \\
Actinobacteria & 0.12 & 0.44 & 0.26 & 0.094 & 0.805 \\
\hline
\end{tabular}

Data are presented as means \pm SEM ( $n=6$; cage was used as experimental unit). $P C$, basal diet + SNE; PA, basal diet extra antibiotics + SNE; PG, basal diet extra $B$. licheniformis + SNE.

TABLE 12 | Effects of $B$. licheniformis and enramycin on relative abundances of genus in cecal microbiota of 25-day-old broilers challenged with SNE (\%).

\begin{tabular}{lccccc}
\hline Items & PC & PA & PG & SEM & P values \\
\hline Faecalibacterium & 11.29 & 10.89 & 17.44 & 2.955 & 0.366 \\
Lactobacillus & 6.38 & 15.43 & 5.75 & 4.093 & 0.414 \\
Barnesiella & 12.68 & 9.45 & 0.17 & 3.304 & 0.116 \\
[Ruminococcus]_torques_group & 5.67 & 5.34 & 8.33 & 1.024 & 0.587 \\
Alistipes & 5.21 & 3.58 & 8.60 & 1.462 & 0.399 \\
Ruminococcaceae_UCG-014 & 5.57 & 4.27 & 6.67 & 0.817 & 0.484 \\
uncultured_bacterium_f_Lachnospiraceae & 3.95 & 4.60 & 5.13 & 0.762 & 0.738 \\
uncultured_bacterium_f_Ruminococcaceae & 5.58 & 4.10 & 3.71 & 0.601 & 0.444 \\
Bacteroides & 4.26 & 7.06 & 0.01 & 1.364 & 0.244 \\
Megamonas & 0.00 & 2.47 & 8.37 & 1.954 & 0.419
\end{tabular}

Data are presented as means \pm SEM ( $n=6$; cage was used as experimental unit). $P C$, basal diet + SNE; PA, basal diet extra antibiotics + SNE; PG, basal diet extra $B$. licheniformis + SNE. Means within a row lacking a common superscript differ significantly $(P<0.05)$.

Alistipes, Ruminococcaceae_UCG-014, uncultured_bacte rium_f_Lachnospiraceae, uncultured_bacterium_f_Rumi-noc occaceae, Bacteroides, and Megamonas. The relative abundance of Others and Unclassified bacterium were 36.0\% (Supplementary Figure 2B). Similarly, no significant differences were detected in abundance of those genera between groups $(P>0.05$, Table 12). Then binary_jaccard algorithm for PERMANOVA/ANOSIM analysis (Beta diversity box plot, Figure 1) was used to evaluate differences in cecal bacterial community structure between different groups. As shown in Figure 1, $\beta$ diversity of the PC group was significantly different from the PA and PG groups; nevertheless, no significant differences were detected in $\beta$ diversity between the PA and PG groups.

LEfSe analysis was used to determine the statistically different biomarkers between groups. As presented in Figure 2, when compared with the PC group, Peptostreptococcaceae, Intestinibacter, and Eisenbergiella were less abundant in the PA group (Figure 2A); nevertheless, Lachnospiraceae_UCG_010 were enriched in the $\mathrm{PG}$ group when compared with group PC (Figure 2B). Furthermore, Clostridiales_vadinBB60_group, g_uncultured_bacterium_f_Clostridiales_vadinBB60_group,

Family_XIII_AD3011_group,

and Ruminococcaceae_NK4A214_group were more abundant in the PG group in contrast to the PA group (Figure 2C).

\section{Predicting the Function of Intestinal Bacteria}

PICRUSt analysis showed a significant functional gene difference between group PC and PA or PG (Figure 3). We found that six pathways were enriched in group PC and eight pathways were in group PA and PG altogether. Besides, 10 pathways were enriched in group PA and 12 pathways in group PA uniquely explaining the fact that the functional profiles representing the microbial communities in group PA and PG were relatively similar and different. Notably, metabolic pathways were mostly common among the significantly differentially represented pathways, which indicated the different metabolic status between groups. Comparing with the group PA, energy metabolism, amino metabolism, and cell motility were enriched in the PC group $(P<0.01$, Figure $3 \mathbf{A})$, whereas carbohydrate metabolism, nucleotide metabolism, xenobiotics biodegradation and metabolism, and membrane transport were significantly enriched in PA group $(P<0.001)$. Compared with the group PG, translation, membrane transport, signal transduction, and cell motility were enriched in the PC group $(P<0.001$, Figure $3 \mathbf{B})$, and metabolism of cofactors and vitamins, biosynthesis of other secondary metabolites, amino acid metabolism, folding sorting and degradation, endocrine system, excretory system, immune system, nervous system, transport, and catabolism were enhanced in group PG $(P<0.001)$.

\section{DISCUSSION}

Necrotic enteritis caused by $C$. perfringens infection destroys the gut integrity of chickens and seriously damages the intestinal function of poultry, leading to a decline in the growth performance (Abudabos et al., 2017). Related studies had shown that probiotics play a positive role on animal health and prevention of NE diseases. Zhou et al. (2016) reported that the body weight decreased significantly after broilers were infected with NE, while FCR increased. However, the improved growth performance was observed when broilers were pre-treated with B. licheniformis H2. Whelan et al. (2018) also demonstrated that Bacillus subtilis DSM32315 alleviated the adverse impact on growth performance of broiler chickens caused by NE infection. Interestingly, Lin et al. (2017) found that diets supplemented with B. licheniformis $\mathrm{H} 2$ which was used in Zhou et al. (2016) research had no significant improvement on body weight, feed intake, and FCR of $C$. perfringens challenged broilers. In accordance with Lin's study, our results showed that pre-treatment with $B$. licheniformis had no improvement on growth performance of SNE-infected broilers. B. licheniformis was believed to prevent $\mathrm{NE}$ in broilers, but the mechanism of action of $B$. licheniformis as a probiotic for the control and prevention of NE is not totally clear. In addition, the function of $B$. licheniformis may differ from different strains and dietary concentrations (Knap et al., 2010). Thus, the optimum application rate of $B$. licheniformis used in studies may need further identified. Furthermore, differences in housing environment, broiler breed, administration route of probiotic $B$. licheniformis as well as the way to establish NE model may influence the outcome of $B$. licheniformis addition 


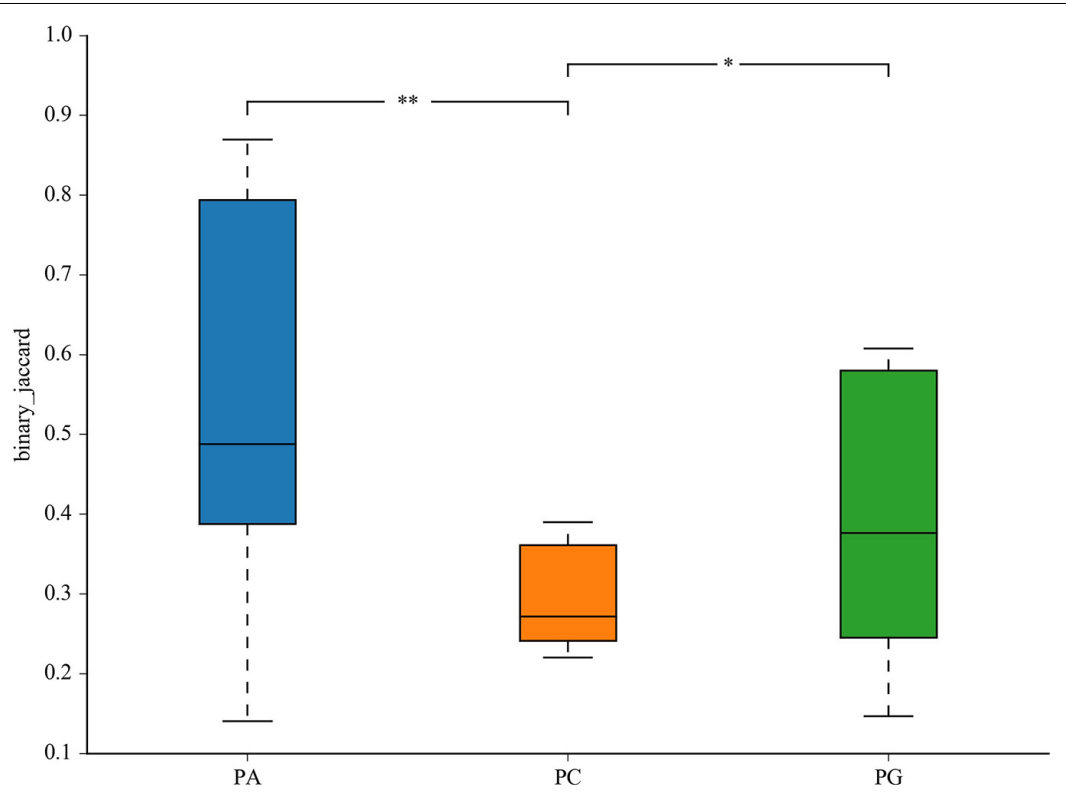

FIGURE 1 | Differential cecum microbiota community ( $\beta$ diversity) between groups in 25-day-old broilers. PC, basal diet $+\mathrm{SNE}$; PA, basal diet extra antibiotics + SNE; PG, basal diet extra B. licheniformis + SNE. Values are means with their standard errors. ${ }^{\star} P<0.05$, ${ }^{\star \star} P<0.01$.

(Ramlucken et al., 2020). However, an improved body weight and FCR of 21-42 days and 1-42 days were seen in the PG and PA group although statistical difference was not reached, indicating that B. licheniformis and enramycin may protect broilers from SNE infection and alleviated growth performance loss caused by SNE to a certain degree. Studies had reported that probiotics Bacillus spp. increased the activity of digestive enzymes such as amylase and protease, and secreted some unknown growthpromoting factors which were helpful for intestinal development, feed degradation, and animal growth (Wang and Gu, 2010; Zhou et al., 2010). This may be one of the potential mechanisms by which probiotics Bacillus spp. improved the growth performance of SNE-infected broilers (Bai et al., 2016).

Intestinal lesion scores, intestinal microbiota balance, bacterial translocation, and intestinal morphology were important indicators for evaluating intestinal integrity and barrier function of broilers. The results of intestinal lesion scores in this study showed that the addition of B. licheniformis significantly reduced the duodenal and total intestinal lesion scores of SNE-infected broilers, which proved that the addition of $B$. licheniformis to diets effectively alleviated intestine damage caused by SNE infection in broilers. Consistent with our results, Wu et al. (2018) reported that B. coagulans significantly reduced intestinal lesion scores of broilers. The results from Jayaraman et al. (2013) also showed that the addition of B. subtilis PB6 significantly decreased the incidence and severity of intestinal lesions in C. perfringens-challenged broilers. In addition, in line with previous studies (Jayaraman et al., 2013; Wang H. et al., 2017; Wu et al., 2018), our results showed that SNE infection led to increased proliferation of cecal C. perfringens and higher $C$. perfringens invasion in the liver, indicating the imbalance of intestinal microflora and barrier damage of intestinal in broilers. After the supplementation of enramycin, lower C. perfringens load in the cecal contents and liver were observed compared with SNE-challenged birds, which proved that enramycin can effectively inhibit the growth of C. perfringens, thereby preventing pathogens or endotoxins from entering the systemic circulation. At 42 days of age, cecal E. coli numbers of broiler chickens in the antibiotics (enramycin) supplement group were significantly higher than that in the other groups. This may mainly be due to the growth inhibiting effect of enramycin on Gram-positive bacteria, especially to the harmful Clostridium in the intestinal tract, thus leading to the mass proliferation of Gram-negative bacteria such as E. coli (Sanjay et al., 2018). The intestinal mucus layer is the first defense barrier dialogued with microorganisms. The mucins secreted by goblet cells are important components of the mucus layer, providing a series of potential recognition sites for intestinal common microorganisms. At the same time, research noted that $C$. perfringens could not synthesize a variety of amino acids (Shimizu et al., 2002) so that the intestinal mucins served as an amino acid source of $C$. perfringens (Collier et al., 2008). In the present study, the addition of enramycin reduced the goblet cells in the jejunum, which may result in a decline in intestinal mucin secretion and a decreased availability of amino acids for $C$. perfringens, thereby inhibiting the growth and proliferation and translocation of $C$. perfringens. The morphological structure and integrity of intestine were associated with growth performance. Consistent with the growth performance that there was only numerically improvement between groups rather than differing significantly, the addition of $B$. licheniformis or enramycin had no significant improvement in the $\mathrm{VH}, \mathrm{CD}$, and $\mathrm{VH} / \mathrm{CD}$ ratios in the jejunum of SNEinfected broilers. Inversely, Jayaraman et al. (2013) reported 

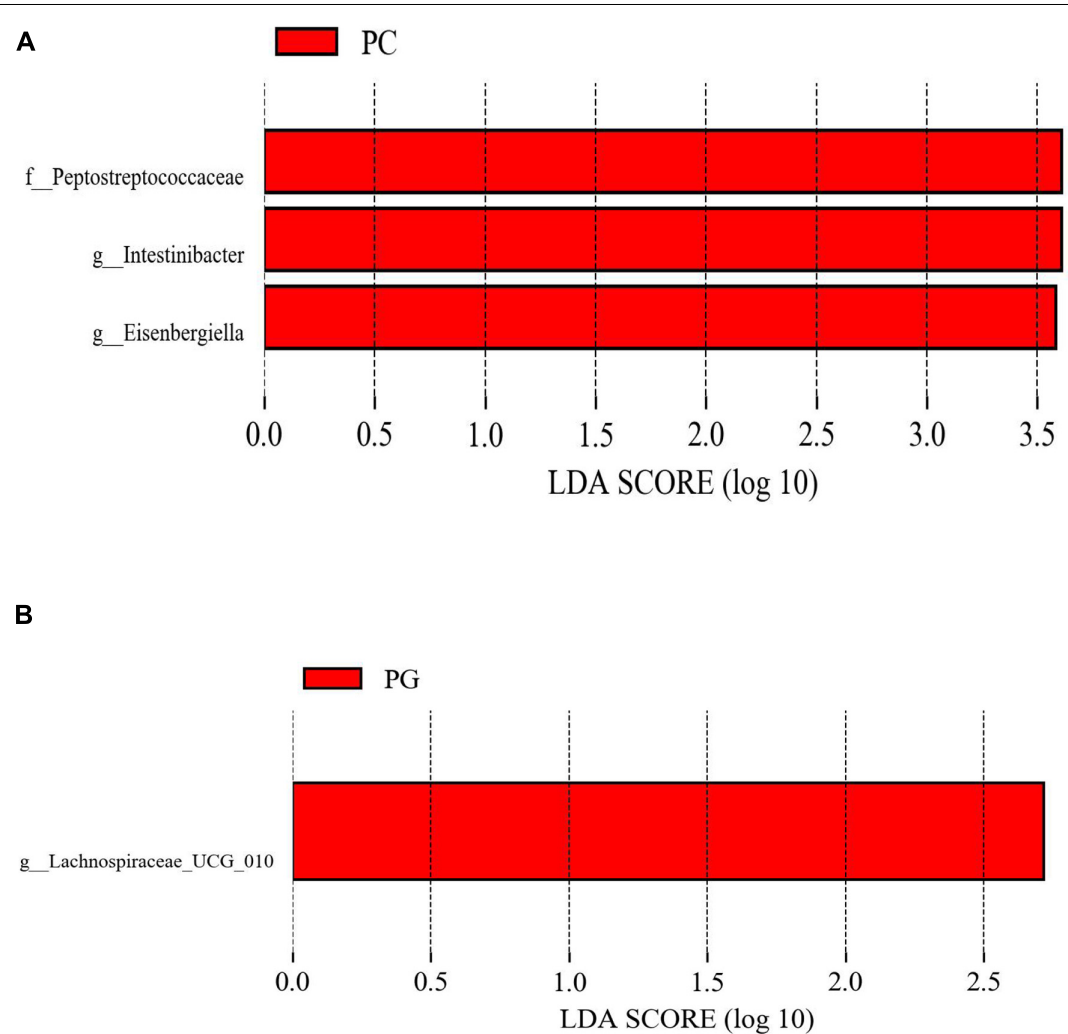

LDA SCORE $(\log 10)$

C

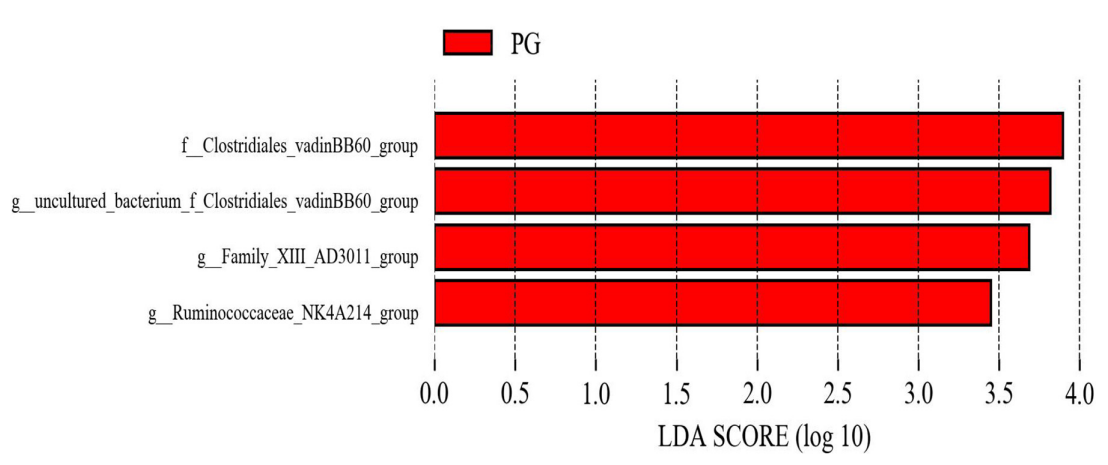

FIGURE 2 | The different phylotypes differed between groups using LEfSe analysis. These figures show the bacteria of which the LDA Score is greater than the set value (the default setting is 2.0) between groups PC and PA. (A) Groups PC and PG. (B) Groups PG and PA. (C) The length of the histogram represents the size of the difference species (i.e., LDA Score), and the different colors represent the different groups. PC, basal diet + SNE; PA, basal diet extra antibiotics + SNE; PG, basal diet extra B. licheniformis + SNE.

that diets added with B. subtilis PB6 significantly increased the intestinal $\mathrm{VH}$ and $\mathrm{VH} / \mathrm{CD}$ ratio of NE-infected broilers, protected the intact villi structure of intestine, and reduced the FCR. As we know that the biofunction of probiotics varies from strain to strain, even the probiotic strains from the same species may work differently (Wang H. et al., 2017). Therefore, the contradictory results may partially be due to the different probiotic strains used in different studies. The aforementioned results also demonstrated that $B$. licheniformis and enramycin could mitigate intestinal injury of SNE-infected broilers in different aspects.

To determine the underlying mechanism responsible for this result, we further investigated the gene expression of tight junction proteins, mucin-2 and TLR signaling pathway related factors, and the alterations in cecal microbiota. The intestinal tight junction (TJ) complex composed of occludins, claudins, ZOs, and other TJ proteins that control the intestinal paracellular permeability facilitates the exchange of water, ions, and other 
A

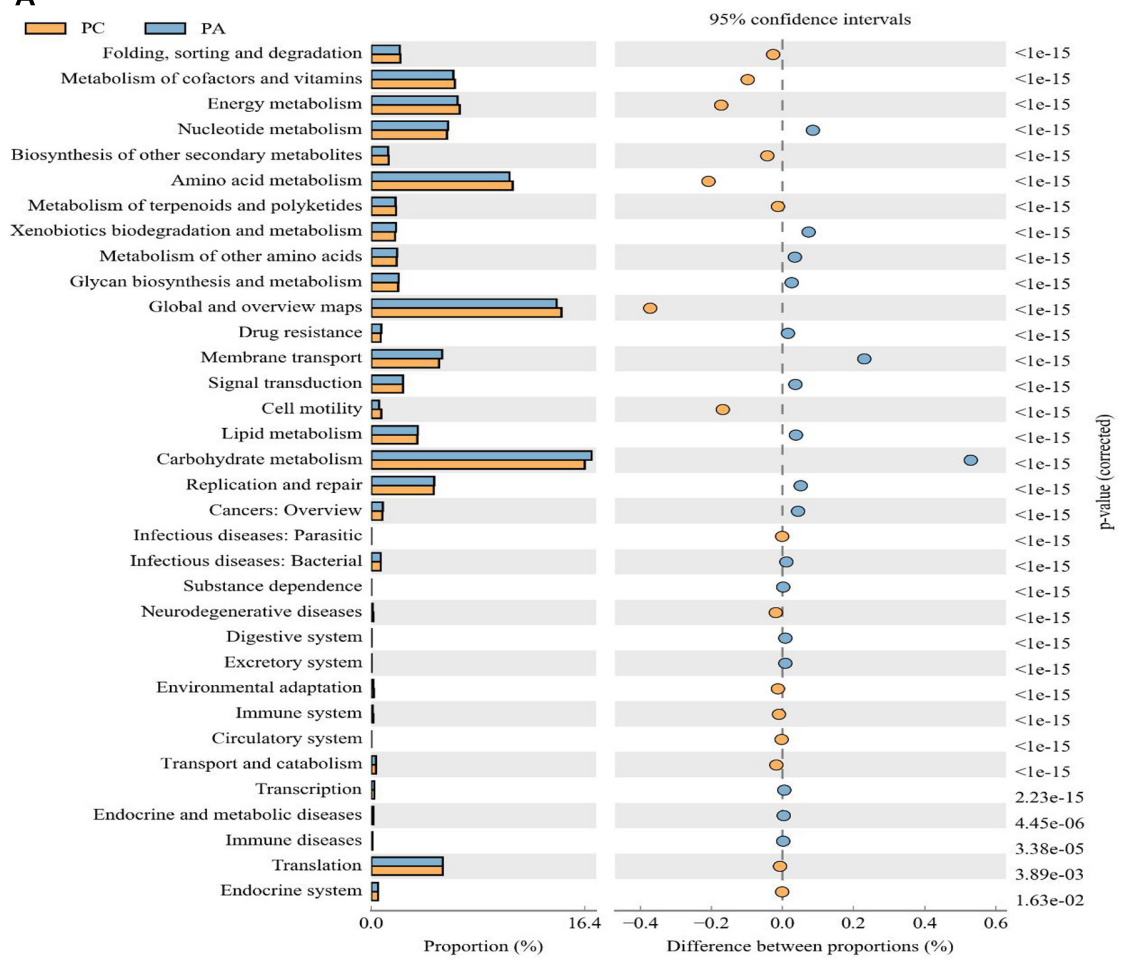

B
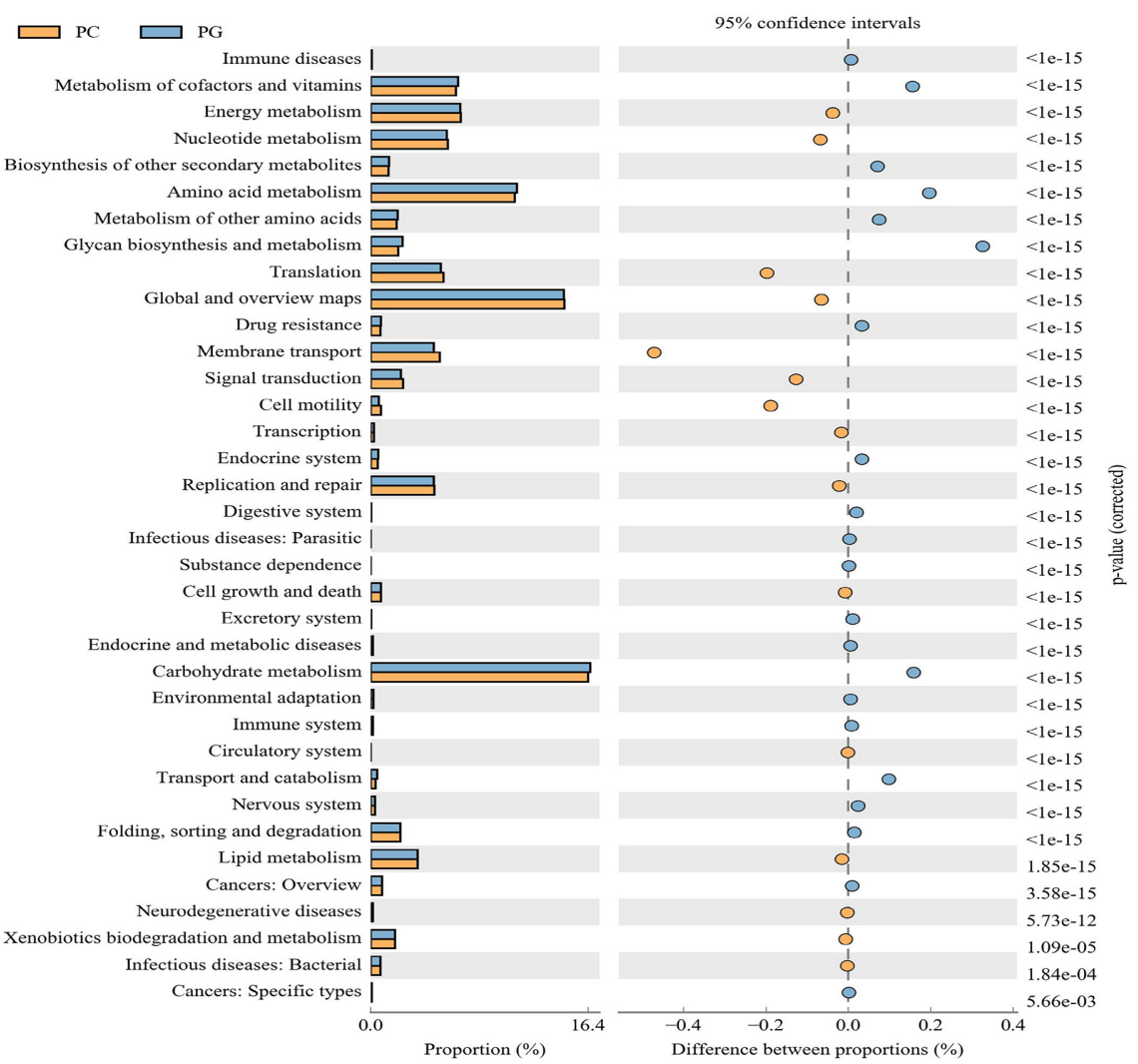

FIGURE 3 | The microbial pathways grouped into level-2 functional categories using PICRUSt. PC between groups PC and PA (A), and groups PC and PG (B). PC, basal diet + SNE; PA, basal diet extra antibiotics + SNE; PG, basal diet extra B. licheniformis + SNE. 
nutrients with external environment, and also plays an important role in resisting the invasion of intestinal pathogens and toxins (Awad et al., 2017). Many pathogens indirectly impair the TJ structures of the intestinal tract by activating the signaling cascades of the host cells (Wageha et al., 2017), while the C. perfringens enterotoxin directly uses the TJ proteins Claudin3 and Claudin-4 as cellular receptors to attach, leading to TJ degradation and increased paracellular permeability (Fujita et al., 2000; Veshnyakova et al., 2010). In the present study, results showed that addition of $B$. licheniformis significantly downregulated the expression of Claudin-3 mRNA in the jejunum of SNE-infected broilers on day 25 and a decreasing trend was also observed in the enramycin group, indicating that $B$. licheniformis and enramycin can reduce the intestinal $C$. perfringens adhesion by down-regulating Claudin-3 mRNA levels, thereby protecting the intestinal mechanical barrier and paracellular permeability. Mucin-2 is one of the mucins which participates in the formation of mucous layer and protects intestinal mucosal barrier integrity (Michael McGuckin et al., 2011). On day 42, C. perfringens loads in the cecal contents of SNE-infected broilers was decreased, and broilers in each group were in the late stage of SNE infection or in the normal health status as can be seen from the results of intestinal lesion scores. At this time point, the gene expressions of Claudin-3 and mucin-2 were significantly up-regulated in the enramycin supplemented group, and the mRNA levels of mucin2 were also increased in the B. licheniformis supplemented group, indicating that $B$. licheniformis and enramycin may convey a protection on the intestinal TJs and mucus layers of broilers (Aliakbarpour et al., 2012; Rajput et al., 2013). In addition, the results also showed that the addition of $B$. licheniformis or enramycin significantly increased the content of cecal formic acid in 42-day-old broilers. Formic acid, a kind of SCFA, is produced by intestinal bacteria fermenting undigested starch or fiber polysaccharides that are unused to the host. SCFAs have important physiological significance to the host (Meimandipour et al., 2010; Rinttila and Apajalahti, 2013), of which formic acid could improve the intestinal morphology of broilers (Garcia et al., 2007) and inhibit the growth of pathogens (Bourassa et al., 2018). The results of the present study showed that B. licheniformis or enramycin can maintain the intestinal health by increasing the content of cecal formic acid in SNE-infected broilers.

When intestinal pathogens invade the host, they can be recognized by pattern recognition receptors. For example, TLRs can identify various pathogen-related molecular patterns and transmit the signals downstream through their linker proteins such as TRIF and $M y D 88$ to activate $N F-\kappa B$, which can be transferred into the nucleus and thus induce the expression of target genes to regulate the immune and inflammation response, cell proliferation, and regeneration (Wertz and Dixit, 2010; Huebener and Schwabe, 2013). In line with other studies (Jung et al., 2015; Rajput et al., 2017), we found that at the peak period of $C$. perfringens infection (2 DPI), adding B. licheniformis or enramycin to the diet significantly increased the jejunal mRNA expression of TRIF and $N F-\kappa B$ in broilers, which indicates the activation of TLR-NF- $\kappa$ B signaling pathway, although the mRNA levels of TLR-4 and TLR-2 were not changed. The TLR-NF- $\mathrm{B}$ signaling pathway is included in the innate immune response.
Its activation causes a series of signal transductions, which leads to the activation and cellular responses of immune-related cells, and subsequently induces the secretion of cytokines, growth factors (TGF- $\beta, I G F-2, G L P-2)$, type I IFNs, and chemokines (Kawai and Akira, 2007). Cytokines are effector molecules that transmit information between immune cells and determine the nature of the immune response at the infection site. For example, $I L-17$ secreted by Th17 cells is an inflammatory cytokine that stimulates the production of granulocytes, promotes the production of antimicrobial peptides by epithelial cells, and enhances innate immunity (Eyerich et al., 2017). Consistent with previous studies (Fasina and Lillehoj, 2018), in this experiment, the addition of enramycin significantly up-regulated the mRNA levels of $I L-17$, indicating that the innate immune function of the broiler intestines was enhanced and effectively resisted the C. perfringens infection. However, unlike previous results (Rajput et al., 2013, 2017; Wang Y. et al., 2017), our results found that $B$. licheniformis did not significantly affect the gene expression of cytokines, such as $I L-1 \beta, I L-10, I L-17$, and TNF- $\alpha$ after activation of TLR-NF- $\mathrm{B}$ signaling pathway. This may be due to the different physiological functions of different probiotic strains (Kang and Sin-Hyeog, 2015). Although there were no significant changes detected on the expression of cytokines, adding $B$. licheniformis significantly up-regulated the expression of growth factors (GLP-2, TGF- $\beta 2$ ), HSP60, HSP70, and HSP90, which was consistent with previous studies (Selvam et al., 2009; Okamoto et al., 2012; Rajput et al., 2017; Tang et al., 2019). Growth factors can promote the cell differentiation, mucosal development, and repair of damaged tissues (Bulut et al., 2008; Massagué, 2012; Camati et al., 2017), while HSPs are anti-stress proteins with molecular chaperone activity that protects cells and tissues from temperature stress or protein denaturation caused by infection, enhancing the resistance to environmental stress (Lindquist and Craig, 1988; Malago et al., 2001). In this experiment, $B$. licheniformis activated the TLR-NF- $\kappa$ B signaling pathway in jejunum of SNE-infected broilers, and afterward upregulating the expression of jejunal growth factors and HSPs, enhancing the ability of tissue repairing and anti-stress of host, but did not affect the gene expression of pro-inflammatory cytokines. Similarly, adding enramycin also activated the TLRNF- $\kappa \mathrm{B}$ signaling pathway in jejunum of 25-day-old SNE-infected broilers, and also increased the gene expression of growth factors (IGF-2, GLP-2, TGF- $\beta 2$ ) and HSP90. Furthermore, the gene expression of the proinflammatory cytokine $I L-17$ was also upregulated in broilers. Therefore, B. licheniformis had an effect of enhancing immunity in contrast to the enramycin. However, the results on day 42 showed that enramycin down-regulated the TLR-NF- $\kappa$ B signaling pathway, the gene expression of proinflammatory cytokines and HSPs in jejunum of healthy broilers, indicating that enramycin decreased the level of immunity and anti-stress of broilers and thus transferred more energy and nutrients to animals for growing. Based on the aforementioned results, we suggested that the impact of $B$. licheniformis on broiler challenged with SNE was focused on the repair and anti-stress of intestine which was different from enramycin's proinflammatory effects although they all activated the TLR-NF- $\kappa$ B signaling pathway. 
Intestinal microbiota affects animal gut development, immune maturation, intestinal barrier, and host susceptibility to pathogens (Sekirov et al., 2010). Therefore, it is important to investigate infection, pre-treatment of $B$. licheniformis, or enramycin on intestinal microflora in broilers infected with SNE. Results showed that there was no significant difference in cecal microbiota $\alpha$-diversity between groups. In accordance with our study, researchers found that $\alpha$-diversity of gut microbiota was not affected by NE infection (Lin et al., 2017; Latorre et al., 2018), antibiotics supplementation (Sanjay et al., 2018), or probiotics Bacillus spp. (Qin et al., 2018) supplementation in broilers. We speculated that C. perfringens infection (Lin et al., 2017), and pre-treatment with antibiotics (Costa et al., 2017; Sanjay et al., 2018) or probiotics Bacillus spp. (Ahmed et al., 2014; Prieto et al., 2014) regulated the proliferation of minor microorganisms in the intestine of broilers thus the $\alpha$-diversity in each group tended to be consistent. In terms of cecal bacterial community structure ( $\beta$ diversity), $B$. licheniformis adding group was similar to the enramycin group, but significantly different from the PC group, indicating that SNE infection caused a disturbance in cecal microflora in broilers, while adding B. licheniformis or enramycin modulated the bacterial community structure. Consistent with our results, Lin et al. (2017) and Xu et al. (2018) demonstrated that $\mathrm{NE}$ infection destroyed the community structure of intestinal microbes in broiler chickens and deviated it from normal state, whereas adding probiotics Bacillus spp. alleviated the disorder of intestinal microflora and restored it into homeostasis.

LEfSe analysis showed that the addition of enramycin reduced the relative abundance of Peptostreptococcaceae, Intestinibacter, and Eisenbergiella in SNE-infected broilers. It was reported that Peptostreptococcaceae were commensal bacteria in the intestine whose proportion in healthy animals was higher than that of diseased animals (Ma et al., 2011; Leng et al., 2016). However, other studies had also reported the presence of opportunistic pathogens in Peptostreptococcaceae may cause host disease (Ma et al., 2011). D'Andreano et al. (2017) compared the jejunal microflora of healthy and hemorrhagic enteritis turkeys, finding that Peptostreptococcaceae were only detected in the jejunum of hemorrhagic enteritis turkeys, which suggested that certain bacteria in Peptostreptococcaceae may also act as harmful bacteria and destroy the intestinal health of the host. For example, Intestinibacter, a genus of Peptostreptococcaceae, was significantly higher in the feces of patients with inflammatory diseases (such as Crohn's disease) than that in healthy individuals (Forbes et al., 2018), which further confirmed our hypothesis. In addition, some studies suggested that the genus Eisenbergiella contains potential pathogenic bacteria (Bernard et al., 2017). Bao et al. (2018) reported that the abundance of Eisenbergiella was significantly increased in the feces of Echinococcus granulosus-infected rats, and speculated that Eisenbergiella might be associated with the host's Th2 immune response. Therefore, in the present study, the enhanced intestinal barrier function and decreased C. perfringens liver translocation of broilers in enramycin group may partially relate to the decreased abundance of Peptostreptococcaceae, Intestinibacter, and Eisenbergiella. Compared with the PC group, we noted that the addition of $B$. licheniformis increased the abundance of Lachnospiraceae_UCG_010 in the cecum of SNE-infected broilers. Researchers reported that the abundance of Lachnospiraceae_UCG_010 was significantly reduced in feces of patients with irritable bowel syndrome, while it was increased in healthy individuals (Zhuang et al., 2018), suggesting that Lachnospiraceae_UCG_010 may be beneficial intestinal bacteria and positively correlate with intestinal health. In this experiment, the increased abundance of Lachnospiraceae_UCG_010 in the B. licheniformis supplement group was consistent with an increase in intestinal barrier function and a decline in intestinal lesion scores. In line with our results, many studies reported that probiotics Bacillus spp. modified the intestinal microflora of NE-infected broilers (Langille et al., 2013; Lin et al., 2017; Xu et al., 2018). Moreover, Clostridiales_vadinBB60_group and one of its genera were enriched in the $B$. licheniformis group when compared with the enramycin group. Clostridiales_vadinBB60_group contains a variety of bacteria producing butyric acid. Studies had reported that the increased abundance of Clostridiales_vadinBB60_group was accompanied by the enhanced serum antioxidant capacity in mice (Shimizu et al., 2002). In addition, Zhang et al. (2018) noted that the presence of Clostridiales_vadinBB60_group was detected in the feces of diabetic rats after treated with liraglutide. It was speculated that Clostridiales_vadinBB60_group may also be beneficial bacteria in intestinal tract, which was good for host health. Ruminococcaceae_NK4A214_group may also be a potentially beneficial bacterium. Studies had demonstrated that the abundance of Ruminococcaceae_NK4A214_group was reduced in obese rats and gout patients (Shao et al., 2017; Zhao et al., 2017). In contrast, Family_XIII_AD3011_group is considered to be a potential pathogen, and many studies reported that the abundance of Family_XIII_AD3011_group is positively associated with the diabetes (Zhang et al., 2018). Our results showed that the addition of $B$. licheniformis increased the abundance of Clostridiales_vadinBB60_group, g_uncultured_bacterium_f_Clostridiales_vadinBB60_group,

Family_XIII_AD3011_group, and Ruminococcaceae_NK4A214_ group were enriched in $B$. licheniformis supplemented group compared with the enramycin group, indicating that the recovery effect of $B$. licheniformis on cecal microbiome disorders of SNE-infected broilers is better than enramycin.

The observed shifts in the intestinal microbiota may regulate gut physiological function, host health, and growth. The PICRUSt aims to predict the unobserved characters from phylogenetic information regarding the organisms in the community. Vitamins are organic compounds which could be produced by bacteria notably vitamin $\mathrm{K}$ and $\mathrm{B}$ groups, regulating the construction and supporting normal physiological function of host (Rowland et al., 2018). An important role it serves is being cofactors for enzymes. Results presented the metabolism of cofactors and vitamins pathway was enriched in group PG, indicating a positive regulative effect of $B$. licheniformis on the activity of enzymatic metabolism (Rozs et al., 2001). However, it was lower in group PA which may result from the antibiotic effect of enramycin. Membrane transport, a vital pathway for the survival of bacteria in the gut ecosystem (Lyons et al., 2017), was increased in group PA, which may point out an 
attempt to compensate for the antibiotic effect of enramycin. Regarding energy metabolism pathway, it was enriched in group PC comparing with group PG and PA, showing an energy metabolites disorder in SNE infection broilers (Chan et al., 2020). Cell motility is the determinant step of pathogen bacteria in early local invasion (Lin et al., 2017). The abundance of cell motility was enriched in PC group as compared with the PA and PG group, indicating the anti-infective effect of $B$. licheniformis and enramycin. In addition, it was found that carbohydrate metabolism pathways were enriched in group PA and PG, according with increased concentration of formic acid in cecum content. As reported, carbohydrate could be metabolized by microflora into SCFAs which were known to boost intestinal health by its trophic and anti-inflammatory effects (Kles and Chang, 2006; Lamas et al., 2018). Qing et al. (2018) noted that SNE infection could affect the hepatic lipid metabolism of chickens and probiotic pretreatment may provide a prophylaxis strategy against SNE infection through regulating lipid metabolism (Zhou et al., 2016; Lin et al., 2017). Agreed with those reports, we found that $B$. licheniformis supplement downregulated the abundance of lipid metabolism pathway; however, enramycin up-regulated it, showing the different regulative effect of B. licheniformis and enramycin on cecum microbial function in the SNE-challenged broilers. Amino acid metabolite polyamines such as putrescine, spermidine, and spermine are harmful to hosts (Stevanato et al., 2012; Bonaiuto et al., 2015). Nevertheless, it was revealed that polyamines support gut physiology by strengthening barrier function, promoting gut maturation, increasing anti-oxidant capacity, and regulating immune function (Lagishetty and Naik, 2008; Bekebrede et al., 2020). As shown that amino acid metabolism pathway was enriched in group PG, this may confirm the hypothesis that $B$. licheniformis could adjust immune function through activating TLR-NF- $\mathrm{B}$ signaling pathway. Therefore, the effect of $B$. licheniformis or enramycin on SNE-challenged broilers needs to be further investigated. Thus, dietary supplementation with antibiotic enramycin and probiotic B. licheniformis affected important predicted functions of the intestinal microbiota of the NE-challenged birds.

\section{CONCLUSION}

Dietary supplementation with B. licheniformis or enramycin mitigated the negative effects of SNE infection in broilers and alleviated intestinal damage, suggesting $B$. licheniformis could be used as an antibiotic alternative. B. licheniformis protected the intestinal health of SNE-infected broilers mainly mediated by increasing the number of beneficial bacteria Lachnospiraceae_UCG_010 and formate acid content in the

\section{REFERENCES}

Abudabos, A. M., Alyemni, A. H., Dafalla, Y. M., and Khan, R. U. (2017). The effect of phytogenics on growth traits, blood biochemical and intestinal histology in cecum, modulating TLR-NF-кB signaling pathway, and upregulating jejunal mucin-2, growth factor (GLP-2 and TGF- $\beta 2$ ), and HSP (HSP60, HSP70, and HSP90) mRNA levels. However, the addition of enramycin maintained the intestinal barrier function mediated by reducing intestinal and liver C. perfringens load, increasing the cecal formate acid concentration, affecting the TLR-NF- $\kappa$ B signaling pathway, and up-regulating intestinal tight junction protein Claudin-3, mucin-2, pro-inflammatory cytokines together with growth factors and HSPs. This study showed that there are similarities and differences on the mechanism of $B$. licheniformis and enramycin in relieving intestinal damage of SNE-infected broilers. More studies are needed to confirm these results in the future.

\section{DATA AVAILABILITY STATEMENT}

The original contributions presented in the study are publicly available. This data can be found here: https://www.ncbi.nlm.nih. gov/, PRJNA728387.

\section{ETHICS STATEMENT}

The animal study was reviewed and approved by China Agricultural University Animal Care and Use Committee (statement no. CAU20170601-2).

\section{AUTHOR CONTRIBUTIONS}

ZW designed the research. LK, YL, and VP performed the experiments and analyzed the data. FG wrote the manuscript. ZW and YG participated in the revision of the manuscript. All authors contributed to data interpretation and approved the final version of the article.

\section{FUNDING}

The authors declare that this study received funding from Chr. Hansen Co., Ltd. (Denmark). The funder was not involved in the study design, collection, analysis, interpretation of data, the writing of this article or the decision to submit it for publication.

\section{SUPPLEMENTARY MATERIAL}

The Supplementary Material for this article can be found online at: https://www.frontiersin.org/articles/10.3389/fmicb. 2021.623739/full\#supplementary-material

broiler chickens exposed to Clostridium perfringens challenge. J. Appl. Anim. Res. 46, 691-695. doi: 10.1080/09712119.2017.1383258

Ahmed, S. T., Islam, M. M., Mun, H. S., Sim, H. J., Kim, Y. J., and Yang, C. J. (2014). Effects of Bacillus amyloliquefaciens as a probiotic strain on growth 
performance, cecal microflora, and fecal noxious gas emissions of broiler chickens. Poult. Sci. 93, 1963-1971. doi: 10.3382/ps.2013-03718

Aliakbarpour, H. R., Chamani, M., Rahimi, G., Sadeghi, A. A., and Qujeq, D. (2012). The Bacillus subtilis and lactic acid bacteria probiotics influences intestinal mucin gene expression, histomorphology and growth performance in broilers. Asian Australas. J. Anim. Sci. 25, 1285-1293. doi: 10.5713/ajas.2012. 12110

Alnassan, A. A., Kotsch, M., Shehata, A. A., Kruger, M., Daugschies, A., and Bangoura, B. (2014). Necrotic enteritis in chickens: development of a straightforward disease model system. Vet. Rec. 174, 455-457. doi: 10.1136/vr. 102066

Awad, W. A., Hess, C., and Hess, M. (2017). Enteric pathogens and their toxininduced disruption of the intestinal barrier through alteration of tight junctions in chickens. Toxins 9:60. doi: 10.3390/toxins 9020060

Bai, K., Huang, Q., Zhang, J., He, J., Zhang, L., and Wang, T. (2016). Supplemental effects of probiotic Bacillus subtilis fmbJ on growth performance, antioxidant capacity, and meat quality of broiler chickens. Poult. Sci. 96, 74-82. doi: 10. $3382 /$ ps/pew246

Baikui, W., Jiangtao, Y., Guoshun, B., Yulong, M., Guoqiao, Y., and Weifen, L. (2016). Effects of Bacillus subtilis B10 on immune function, antioxidant indices and serum biochemical parameters of broilers. Feed Ind. 37, 47-51. doi: 10.13302/j.cnki.fi.2016.17.011

Bao, J., Zheng, H., Wang, Y., Zheng, X., He, L., Qi, W., et al. (2018). Echinococcus granulosus infection results in an increase in Eisenbergiella and Parabacteroides genera in the gut of mice. Front. Microbiol. 9:2890. doi: 10.3389/fmicb.2018. 02890

Bekebrede, A., Keijer, J., Gerrits, W., and Boer, V. (2020). The molecular and physiological effects of protein-derived polyamines in the intestine. Nutrients 12:197. doi: 10.3390/nu12010197

Bernard, K., Burdz, T., Wiebe, D., Balcewich, B. M., Zimmerman, T., Lagacé-Wiens, P., et al. (2017). Characterization of isolates of Eisenbergiella tayi, a strictly anaerobic Gram-stain variable bacillus recovered from human clinical materials in Canada. Anaerobe 44, 128-132. doi: 10.1016/j.anaerobe.2017.03.005

Bonaiuto, E., Grancara, S., Martinis, P., Stringaro, A., and Paolo, M. L. D. (2015). A novel enzyme with spermine oxidase properties in bovine liver mitochondria: identification and kinetic characterization. Free Radic. Biol. Med. 81, 88-99. doi: 10.1016/j.freeradbiomed.2015.01.001

Bourassa, D. V., Wilson, K. M., Ritz, C. R., Kiepper, B. K., and Buhr, R. J. (2018). Evaluation of the addition of organic acids in the feed and/or water for broilers and the subsequent recovery of Salmonella typhimurium from litter and ceca. Poult. Sci. 97, 64-73. doi: 10.3382/ps/pex289

Bulut, K., Pennartz, C., Felderbauer, P., Meier, J. J., Banasch, M., Bulut, D., et al. (2008). Glucagon like peptide-2 induces intestinal restitution through VEGF release from subepithelial myofibroblasts. Eur. J. Pharmacol. 578, 279-285. doi: 10.1016/j.ejphar.2007.08.044

Camati, P. R., Giovanini, A. F., de Miranda Peixoto, H. E., Schuanka, C. M., Giacomel, M. C., de Araújo, M. R., et al. (2017). Immunoexpression of IGF1, IGF2, and osteopontin in craniofacial bone repair associated with autogenous grafting in rat models treated with alendronate sodium. Clin. Oral Investig. 21, 1895-1903. doi: 10.1007/s00784-016-1975-0

Caporaso, J. G., Kuczynski, J., Stombaugh, J., Bittinger, K., Bushman, F. D., Costello, E. K., et al. (2010). QIIME allows analysis of high-throughput community sequencing data. Nat. Methods 7, 335-336. doi: 10.1038/nmeth.f. 303

Chan, S. Y., Probert, F., Radford-Smith, D. E., Hebert, J. C., Claridge, T. D. W., Anthony, D. C., et al. (2020). Post-inflammatory behavioural despair in male mice is associated with reduced cortical glutamate-glutamine ratios, and circulating lipid and energy metabolites. Sci. Rep. 10:16857. doi: 10.1038/ s41598-020-74008-w

Collier, C. T., Hofacre, C. L., Payne, A. M., Anderson, D. B., Kaiser, P., Mackie, R. I., et al. (2008). Coccidia-induced mucogenesis promotes the onset of necrotic enteritis by supporting Clostridium perfringens growth. Vet. Immunol. Immunopathol. 122, 104-115. doi: 10.1016/j.vetimm.2007.10.014

Costa, M. C., Bessegatto, J. A., Alfieri, A. A., Weese, J. S., and Oba, A. (2017). Different antibiotic growth promoters induce specific changes in the cecal microbiota membership of broiler chicken. PLoS One 12:e0171642. doi: 10. 1371/journal.pone.0171642
D’Andreano, S., Sànchez Bonastre, A., Francino, O., Cuscó Martí, A., Lecchi, C., Grilli, G., et al. (2017). Gastrointestinal microbial population of turkey (Meleagris gallopavo) affected by hemorrhagic enteritis virus. Poult. Sci. 96, 3550-3558. doi: 10.3382/ps/pex139

Edgar, R. C. (2010). Search and clustering orders of magnitude faster than BLAST. Bioinformatics 26, 2460-2461. doi: 10.1093/bioinformatics/btq461

Engström, B. E., Fermér, C., Lindberg, A., Saarinen, E., Båverud, V., and Gunnarsson, A. (2003). Molecular typing of isolates of Clostridium perfringens from healthy and diseased poultry. Vet. Microbiol. 94, 225-235. doi: 10.1016/ S0378-1135(03)00106-8

Eyerich, K., Dimartino, V., and Cavani, A. (2017). IL-17 and IL-22 in immunity: driving protection and pathology. Eur. J. Immunol. 47, 607-614. doi: 10.1002/ eji.201646723

Fasina, Y. O., and Lillehoj, H. S. (2018). Characterization of intestinal immune response to Clostridium perfringens infection in broiler chickens. Poult. Sci. 98, 188-198. doi: 10.3382/ps/pey390

Forbes, J. D., Chen, C. Y., Knox, N. C., Marrie, R. A., El-Gabalawy, H., De Kievit, T., et al. (2018). A comparative study of the gut microbiota in immune-mediated inflammatory diseases - Does a common dysbiosis exist? Microbiome 6:221. doi: 10.1186/s40168-018-0603-4

Forder, R., Nattrass, G. S., Geier, M. S., Hughes, R. J., and Hynd, P. I. (2012). Quantitative analyses of genes associated with mucin synthesis of broiler chickens with induced necrotic enteritis. Poult. Sci. 91, 1335-1341. doi: 10.3382/ ps.2011-02062

Fujita, K., Katahira, J., Horiguchi, Y., Sonoda, N., Furuse, M., and Tsukita, S. (2000). Clostridium perfringens enterotoxin binds to the second extracellular loop of claudin-3, a tight junction integral membrane protein. FEBS Lett. 476, 258-261. doi: 10.1016/S0014-5793(00)01744-0

Garcia, V., Catala-Gregori, P., Hernandez, F., Megias, M. D., and Madrid, J. (2007). Effect of formic acid and plant extracts on growth, nutrient digestibility, intestine mucosa morphology, and meat yield of broilers. J. Appl. Poultry Res. 16, 555-562. doi: 10.3382/japr.2006-00116

Gholamiandehkordi, A. R., Timbermont, L., Lanckriet, A., Van Den Broeck, W., Pedersen, K., Dewulf, J., et al. (2007). Quantification of gut lesions in a subclinical necrotic enteritis model. Avian Pathol. 36, 375-382. doi: 10.1080/ 03079450701589118

Golder, H. M., Geier, M. S., Forder, R. E. A., Hynd, P. I., and Hughes, R. J. (2011). Effects of necrotic enteritis challenge on intestinal micro-architecture and mucin profile. Br. Poult. Sci. 52, 500-506. doi: 10.1080/00071668.2011.5 87183

Guo, S., Liu, D., Zhao, X., Li, C., and Guo, Y. (2014). Xylanase supplementation of a wheat-based diet improved nutrient digestion and mRNA expression of intestinal nutrient transporters in broiler chickens infected with Clostridium perfringens. Poult. Sci. 93, 94-103. doi: 10.3382/ps.2013-03188

Huebener, P., and Schwabe, R. F. (2013). Regulation of wound healing and organ fibrosis by toll-like receptors. Biochim. Biophys. Acta 1832, 1005-1017. doi: 10.1016/j.bbadis.2012.11.017

Jayaraman, S., Thangavel, G., Kurian, H., Mani, R., Mukkalil, R., and Chirakkal, H. (2013). Bacillus subtilis PB6 improves intestinal health of broiler chickens challenged with Clostridium perfringens-induced necrotic enteritis. Poult. Sci. 92, 370-374. doi: 10.3382/ps.2012-02528

Jung, J. U., Shin, J. U., Rhee, Y. K., Cho, C. O., and Lee, K. A. (2015). In vitro and in vivo immunostimulatory activity of an exopolysaccharide-enriched fraction from Bacillus subtilis. J. Appl. Microbiol. 118, 739-752. doi: 10.1111/jam.12742

Kang, H. J., and Sin-Hyeog, I. M. (2015). Probiotics as an immune modulator. J. Nutr. Sci. Vitaminol. 61, S103-S105. doi: 10.3177/jnsv.61.S103

Kawai, T., and Akira, S. (2007). Signaling to NF-кB by Toll-like receptors. Trends Mol. Med. 13, 460-469. doi: 10.1016/j.molmed.2007.09.002

Khan, R. U., and Naz, S. (2013). Application of probiotics in poultry production. Worlds Poult. Sci. J. 69, 621-632. doi: 10.1017/S0043933913000627

Kim, Y., Cho, J. Y., Kuk, J. H., Moon, J. H., Cho, J. I., Kim, Y. C., et al. (2004). Identification and antimicrobial activity of phenylacetic acid produced by Bacillus licheniformis isolated from fermented soybean, Chungkook-Jang. Curr. Microbiol. 48, 312-317. doi: 10.1007/s00284-003-4193-3

Kles, K. A., and Chang, E. B. (2006). Short-chain fatty acids impact on intestinal adaptation, inflammation, carcinoma, and failure. Gastroenterology 130, S100S105. doi: 10.1053/j.gastro.2005.11.048 
Knap, I., Lund, B., Kehlet, A. B., Hofacre, C., and Mathis, G. (2010). Bacillus licheniformis prevents necrotic enteritis in broiler Chickens. Avian Dis. 54, 931-935. doi: 10.1637/9106-101509-ResNote.1

Lagishetty, C. V., and Naik, S. R. (2008). Polyamines: potential anti-inflammatory agents and their possible mechanism of action. Indian J. Pharmacol. 40, 121125. doi: 10.4103/0253-7613.42305

Lamas, B., Natividad, J. M., and Sokol, H. (2018). Aryl hydrocarbon receptor and intestinal immunity. Mucosal Immunol. 11, 1024-1038. doi: 10.1038/s41385018-0019-2

Langille, M. G. I., Zaneveld, J., Caporaso, J. G., McDonald, D., Knights, D., Reyes, J. A., et al. (2013). Predictive functional profiling of microbial communities using 16S rRNA marker gene sequences. Nat. Biotechnol. 31, 814-821. doi: 10.1038/nbt.2676

Latorre, J. D., Bishnu, A., Park, S. H., Teague, K. D., Graham, L. E., Mahaffey, B. D., et al. (2018). Evaluation of the epithelial barrier function and Ileal microbiome in an established necrotic enteritis challenge model in broiler Chickens. Front. Vet. Sci. 5:199. doi: 10.3389/fvets.2018.00199

Lee, K. W., Lillehoj, H. S., Jeong, W., Jeoung, H. Y., and An, D. J. (2011). Avian necrotic enteritis: experimental models, host immunity, pathogenesis, risk factors, and vaccine development. Poult. Sci. 90, 1381-1390. doi: 10.3382/ ps.2010-01319

Leng, Y., Yi, M., Fan, J., Bai, Y., Ge, Q., and Yao, G. (2016). Effects of acute intraabdominal hypertension on multiple intestinal barrier functions in rats. Sci. Rep. 6:22814. doi: 10.1038/srep22814

Lin, Y., Xu, S., Zeng, D., Ni, X., Zhou, M., Zeng, Y., et al. (2017). Disruption in the cecal microbiota of chickens challenged with Clostridium perfringens and other factors was alleviated by Bacillus licheniformis supplementation. PLoS One 12:e0182426. doi: 10.1371/journal.pone.0182426

Lindquist, S., and Craig, E. A. (1988). The heat-shock proteins. Annu. Rev. Genet. 22, 631-677. doi: 10.1146/annurev.ge.22.120188.003215

Livak, K. J., and Schmittgen, T. D. (2001). Analysis of relative gene expression data using real-time quantitative PCR and the 2(-Delta Delta C(T)) Method. Methods 25, 402-408. doi: 10.1006/meth.2001

Lyons, P. P., Turnbull, J. F., Dawson, K. A., and Crumlish, M. (2017). Phylogenetic and functional characterization of the distal intestinal microbiome of rainbow trout Oncorhynchus mykiss from both farm and aquarium settings. J. Appl. Microbiol. 12, 347-363. doi: 10.1111/jam.13347

Ma, C., Wu, X., Nawaz, M., Li, J., Yu, P., Moore, J. E., et al. (2011). Molecular characterization of fecal microbiota in patients with viral Diarrhea. Curr. Microbiol. 63, 259-266. doi: 10.1007/s00284-011-9972-7

Malago, J. J., Koninkx, J. F. J. G., and Dijk, J. E. V. (2001). BioOne Online Journals - The heat shock response and cytoprotection of the intestinal epithelium. Cell Stress Chaperon 10, 4-6. doi: 10.1379/CSC-91.1

Massagué, J. (2012). TGFß signalling in context. Nat. Rev. Mol. Cell Biol. 13, 616-630. doi: 10.1016/j.ejphar.2007.08.044

Meimandipour, A., Shuhaimi, M., Soleimani, A. F., Azhar, K., Hair-Bejo, M., Kabeir, B. M., et al. (2010). Selected microbial groups and short-chain fatty acids profile in a simulated chicken cecum supplemented with two strains of Lactobacillus. Poult. Sci. 89, 470-476. doi: 10.3382/ps.2009-00495

Michael McGuckin, M. A., Lindén, S. K., Sutton, P., and Florin, T. H. (2011). Mucin dynamics and enteric pathogens. Nat. Rev. Microbiol. 9, 265-278. doi: $10.1038 /$ nrmicro2538

Mingmongkolchai, S., and Panbangred, W. (2018). Bacillus probiotics: an alternative to antibiotics for livestock production. J. Appl. Microbiol. 124, 1334-1346. doi: 10.1111/jam.13690

Mishra, N., and Smyth, J. A. (2017). Oral vaccination of broiler chickens against necrotic enteritis using a non-virulent NetB positive strain of Clostridium perfringens type A. Vaccine 32, 6858-6865. doi: 10.1016/j.vaccine.2017.10.030

National Research Council (1994). Nutrient Requirements of Poultry 9th Edn, Washington, DC: National Academies Press.

Okamoto, K., Fujiya, M., and Nata, T. (2012). W1835 competence and sporulation factor derived from Bacillus Subtilis improves epithelial cell injury in intestinal inflammation via immunomodulation and cytoprotection. Int. J. Colorectal Dis. 27, 1039-1046. doi: 10.1016/S0016-5085(10)63455-5

Parish, W. E. (1961). Necrotic enteritis in the fowl (Gallus gallus domesticus). I. Histopathology of the disease and isolation of a strain of Clostridium welchii. J. Comp. Pathol. 71, 377-393. doi: 10.1016/S0368-1742(61)80043-X
Park, S. S., Lillehoj, H. S., Allen, P. C., Park, D. W., Fitzcoy, S., Bautista, D. A., et al. (2008). Immunopathology and cytokine responses in broiler chickens coinfected with Eimeria maxima and Clostridium perfringens with the use of an animal model of necrotic enteritis. Avian Dis. 52, 14-22. doi: 10.1637/7997041707-Reg

Prescott, J. F., Smyth, J. A., Shojadoost, B., and Vince, A. (2016). Experimental reproduction of necrotic enteritis in chickens: a review. Avian Pathol. 45, 317-322. doi: 10.1080/03079457.2016.1141345

Prieto, M. L., O’Sullivan, L., Tan, S. P., McLoughlin, P., Hughes, H., Gutierrez, M., et al. (2014). In vitro assessment of marine bacillus for use as livestock probiotics. Mar. Drugs 12, 2422-2445. doi: 10.3390/md12052422

Qin, C., Gong, L., Zhang, X., Wang, Y., Wang, Y., Wang, B., et al. (2018). Effect of Saccharomyces boulardii and Bacillus subtilis B10 on gut microbiota modulation in broilers. Anim. Nutr. 4, 358-366.

Qing, X., Zeng, D., Wang, H., Ni, X., and Jing, B. (2018). Analysis of hepatic transcriptome demonstrates altered lipid metabolism following Lactobacillus johnsonii BS15 prevention in chickens with subclinical necrotic enteritis. Lipids Health Dis. 17:93. doi: 10.1186/s12944-018-0741-5

Rajput, I. R., Li, L. Y., Xin, X., Wu, B. B., Juan, Z. L., Cui, Z. W., et al. (2013). Effect of Saccharomyces boulardii and Bacillus subtilis B10 on intestinal ultrastructure modulation and mucosal immunity development mechanism in broiler chickens. Poult. Sci. 92, 956-965. doi: 10.3382/ps.2012-02845

Rajput, I. R., Ying, H., Yajing, S., Arain, M. A., and Wenhua, L. (2017). Correction: Saccharomyces boulardii and Bacillus Subtilis B10 modulate TLRs and cytokines expression patterns in jejunum and ileum of broilers. PLoS One 12:e173917. doi: 10.1371/journal.pone.0173917

Ramlucken, U., Lalloo, R., Roets, Y., Moonsamy, G., and Thantsha, M. S. (2020). Advantages of bacillus based probiotics in poultry production. Livest. Sci. 241:104215. doi: 10.1016/j.livsci.2020.104215

Rinttila, T., and Apajalahti, J. (2013). Intestinal microbiota and metabolitesImplications for broiler chicken health and performance. J. Appl. Poult. Res. 22, 647-658. doi: 10.3382/japr.2013-00742

Rowland, I., Gibson, G., Heinken, A., Scott, K., Swann, J., Thiele, I., et al. (2018). Gut microbiota functions: metabolism of nutrients and other food components. Eur. J. Nutr. 57, 1-24. doi: 10.1007/s00394-017-1445-8

Rozs, M., Manczinger, L., Cs, V., and Kevei, F. (2001). Secretion of a trypsinlike thiol protease by a new keratinolytic strain of Bacillus licheniformis. FEMS Microbiol. Lett. 205, 221-224. doi: 10.1016/S0378-1097(01)00462-1

Sanjay, K., Chongxiao, C., Nagaraju, I., Orosco, W. G., Manpreet, S., Kyun, K. W., et al. (2018). Effect of antibiotic withdrawal in feed on chicken gut microbial dynamics, immunity, growth performance and prevalence of foodborne pathogens. PLoS One 13:e0192450. doi: 10.1371/journal.pone.0192450

Segata, N., Izard, J., Waldron, L., Gevers, D., Miropolsky, L., Garrett, W. S., et al. (2011). Metagenomic biomarker discovery and explanation. Genome Biol. 12:R60. doi: 10.1186/gb-2011-12-6-r60

Sekirov, I., Russell, S. L., Antunes, L. C. M., and Finlay, B. B. (2010). Gut microbiota in health and disease. Physiol. Rev. 71, 242-246. doi: 10.1152/physrev.00045. 2009

Selvam, R., Maheswari, P., Kavitha, P., Ravichandran, M., Sas, B., and Ramchand, C. N. (2009). Effect of Bacillus subtilis PB6, a natural probiotic on colon mucosal inflammation and plasma cytokines levels in inflammatory bowel disease. Indian J. Biochem. Biophys. 46, 79-85. doi: 10.1007/s00249-008-0372-2

Shao, T., Li, S., Li, H., Xie, Z., He, Z., and Wen, C. (2017). Combined signature of the fecal microbiome and metabolome in patients with gout. Front. Microbiol. 8:268. doi: 10.3389/fmicb.2017.00268

Shimizu, T., Ohtani, K., Hirakawa, H., Ohshima, K., Yamashita, A., Shiba, T., et al. (2002). Complete genome sequence of Clostridium perfringens, an anaerobic flesh-eater. Proc. Natl. Acad. Sci. U.S.A. 99, 996-1001. doi: 10.1073/pnas. 022493799

Song, B., Li, H., Wu, Y., Zhen, W., and Guo, Y. (2017). Effect of microencapsulated sodium butyrate dietary supplementation on growth performance and intestinal barrier function of broiler chickens infected with necrotic enteritis. Anim. Feed Sci. Technol. 232, 6-15. doi: 10.1016/j.anifeedsci.2017.07.009

Stevanato, R., Cardillo, S., Braga, M., Iuliis, A. D., Battaglia, V., Toninello, A., et al. (2012). Preliminary kinetic characterization of a copper amine oxidase from rat liver mitochondria matrix. Amino Acids 42, 2531-2531. doi: 10.1007/s00726012-1227-9 
Tang, W., Qian, Y., Yu, B., Zhang, T., Gao, J., He, J., et al. (2019). Effects of Bacillus subtilis DSM32315 supplementation and dietary crude protein level on performance, gut barrier function and microbiota profile in weaned piglets. J. Anim. Sci. 97, 2125-2138. doi: 10.1093/jas/skz090

Tian, X., Shao, Y., Guo, Y., and Wang, Z. (2016). Effects of dietary yeast betaglucans supplementation on growth performance, gut morphology, intestinal Clostridium perfringens population and immune response of broiler chickens challenged with necrotic enteritis. Anim. Feed Sci. Technol. 215, 144-155. doi: 10.1016/j.anifeedsci.2016.03.009

Timbermont, L., Haesebrouck, F., Ducatelle, R., and Van Immerseel, F. (2011). Necrotic enteritis in broilers: an updated review on the pathogenesis. Avian Pathol. 40, 341-347. doi: 10.1080/03079457.590967

Venessa, E., Wang, J., Alexander, V. P., Freddy, H., Marie, J., Gwen, F., et al. (2016). The probiotic Butyricicoccus pullicaecorum reduces feed conversion and protects from potentially harmful intestinal microorganisms and necrotic enteritis in broilers. Front. Microbiol. 7:1416. doi: 10.3389/fmicb.2016.01416

Veshnyakova, A., Protze, J., Rossa, J., Blasig, I. E., Krause, G., and Piontek, J. (2010). On the interaction of Clostridium perfringens Enterotoxin with Claudins. Toxins 2, 1336-1356. doi: 10.3390/toxins2061336

Wade, B., and Keyburn, A. (2015). The true cost of necrotic enteritis. World Poult. Sci. J. 31, 16-17.

Wageha, A., Claudia, H., and Michael, H. (2017). Enteric pathogens and their toxin-induced disruption of the intestinal barrier through alteration of tight junctions in chickens. Toxins 9:60.

Wang, H., Ni, X., Xiaodan, Q., Lei, L., Jing, L., Abdul, K., et al. (2017). Probiotic enhanced intestinal immunity in broilers against subclinical necrotic enteritis. Front. Microbiol. 8:1592. doi: 10.3389/fimmu.2017.01592

Wang, Y., Du, W., Lei, K., Wang, B., Wang, Y., Zhou, Y., et al. (2017). Effects of dietary Bacillus licheniformis on gut physical barrier, immunity, and reproductive hormones of laying hens. Probiotics Antimicrob Proteins 9, 292-299. doi: 10.1007/s12602-017-9252-3

Wang, Y., and Gu, Q. (2010). Effect of probiotic on growth performance and digestive enzyme activity of Arbor Acres broilers. Res. Vet. Sci. 89, 163-167. doi: 10.1016/j.rvsc.2010.03.009

Wertz, I. E., and Dixit, V. M. (2010). Signaling to NF-кB: regulation by ubiquitination. Cold Spring Harb. Perspect. Biol. 2:a003350. doi: 10.1101/ cshperspect.a003350

Whelan, R. A., Doranalli, K., Rinttilä, T., Vienola, K., Jurgens, G., and Apajalahti, J. (2018). The impact of Bacillus subtilis DSM 32315 on the pathology, performance, and intestinal microbiome of broiler chickens in a necrotic enteritis challenge. Poult. Sci. 98, 3450-3463. doi: 10.3382/ps/pey500
Wu, Y., Shao, Y., Song, B., Zhen, W., Wang, Z., Guo, Y., et al. (2018). Effects of Bacillus coagulans supplementation on the growth performance and gut health of broiler chickens with Clostridium perfringens-induced necrotic enteritis. J. Anim. Sci. Biotechnol. 9:9. doi: 10.1186/s40104-017-0220-2

Xu, S., Lin, Y., Zeng, D., Zhou, M., Zeng, Y., Wang, H., et al. (2018). Bacillus licheniformis normalize the ileum microbiota of chickens infected with necrotic enteritis. Sci. Rep. 8:1744. doi: 10.1038/s41598-018-20059-z

Yin, D., Du, E., Yuan, J., Gao, J., Wang, Y. L., Aggrey, S. E., et al. (2017). Supplemental thymol and carvacrol increases ileum Lactobacillus population and reduces effect of necrotic enteritis caused by Clostridium perfringes in chickens. Sci. Rep. 7:7334. doi: 10.1038/s41598-017-07420-4

Zhang, Q., Xiao, X., Zheng, J., Li, M., Yu, M., Ping, F., et al. (2018). Featured article: structure moderation of gut microbiota in liraglutidetreated diabetic male rats. Exp. Biol. Med. 243, 34-44. doi: 10.1177/15353702 17743765

Zhao, L., Zhang, Q., Ma, W., Tian, F., Shen, H., and Zhou, M. (2017). A Combination of quercetin and resveratrol reduces obesity in high-fat diet-fed rats by modulation of gut microbiota. Food Funct. 8, 4644-4656. doi: 10.1039/ C7FO01383C

Zhou, M., Zeng, D., Ni, X., Tu, T., Yin, Z., Pan, K., et al. (2016). Effects of Bacillus licheniformis on the growth performance and expression of lipid metabolismrelated genes in broiler chickens challenged with Clostridium perfringensinduced necrotic enteritis. Lipids Health Dis. 15:48. doi: 10.1186/s12944-0160219-2

Zhou, X., Wang, Y., Gu, Q., and Li, W. (2010). Effect of dietary probiotic, Bacillus coagulans, on growth performance, chemical composition, and meat quality of Guangxi Yellow chicken. Poult. Sci. 89, 588-593. doi: 10.3382/ps.2009-00319

Zhuang, X., Tian, Z., Li, L., Zeng, Z., Chen, M., and Xiong, L. (2018). Fecal microbiota alterations associated with diarrhea-predominant irritable bowel syndrome. Front. Microbiol. 9:1600. doi: 10.3389/fmicb.2018.01600

Conflict of Interest: The authors declare that the research was conducted in the absence of any commercial or financial relationships that could be construed as a potential conflict of interest.

Copyright (c) 2021 Kan, Guo, Liu, Pham, Guo and Wang. This is an open-access article distributed under the terms of the Creative Commons Attribution License (CC BY). The use, distribution or reproduction in other forums is permitted, provided the original author(s) and the copyright owner(s) are credited and that the original publication in this journal is cited, in accordance with accepted academic practice. No use, distribution or reproduction is permitted which does not comply with these terms. 